\title{
On the stabilization parameter in the subgrid scale approximation of scalar convection-diffusion-reaction equations on distorted meshes
}

\author{
Javier Principe ${ }^{\mathrm{a}, *}$, Ramon Codina ${ }^{\mathrm{b}}$ \\ ${ }^{a}$ International Center for Numerical Methods in Engineering (CIMNE), Jordi Girona 1-3, Edifici C1, 08034 Barcelona, Spain \\ ${ }^{\mathrm{b}}$ Universitat Politècnica de Catalunya, Jordi Girona 1-3, Edifici C1, 08034 Barcelona, Spain
}

\section{A R T I C L E I N F O}

\section{Article history:}

Received 18 March 2009

Accepted 1 August 2009

Available online 15 August 2009

\begin{abstract}
A B S T R A C T
In this paper we revisit the definition of the stabilization parameter in the finite element approximation of the convection-diffusion-reaction equation. The starting point is the decomposition of the unknown into its finite element component and a subgrid scale that needs to be approximated. In order to incorporate the distortion of the mesh into this approximation, we transform the equation for the subgrid scale within each element to the shape-regular reference domain. The expression for the subgrid scale arises from an approximate Fourier analysis and the identification of the wave number direction where instabilities are most likely to occur. The final outcome is an expression for the stabilization parameter that accounts for anisotropy and the dominance of either convection or reaction terms in the equation.
\end{abstract}

(c) 2009 Elsevier B.V. All rights reserved.

\section{Introduction}

When attempting the numerical solution of the convection-diffusion-reaction (CDR) equation, the first problem identified is the lack of stability of the Galerkin formulation when the convective term is important, which manifests as numerical oscillations that pollute the solution in the whole domain, and particularly near boundary layers. After understanding this problem as a lack of diffusion in the discrete problem, the first solution was to add numerical dissipation, developing upwind techniques in the context of the finite difference method. The inconsistent extra terms implied a loss of accuracy and the situation was fixed with the introduction of the SUPG method in $[38,43,8]$, which was analyzed in [42]. This method depends on the so-called stabilization parameter, usually denoted by $\tau$. This parameter is also present in the Galerkin least squares method (GLS), introduced in [40] and analyzed in [25] as well as in the Douglas-Wang method introduced in [21] in the context of the Stokes problem. These methods were related to the introduction of bubble functions in $[5,3,7,24]$, where it was shown that a choice of the bubble implies a choice of the stabilization parameter. The optimal bubble is given by the solution of a local subproblem driven by the residual [29], therefore named residual free. A general approach to the development of stabilized formulations is the variational multiscale method (VMM) introduced in $[37,39]$, based on a decomposition of the space into a coarse scale resolvable part and a fine scale subgrid part that, after some

\footnotetext{
* Corresponding author.

E-mail addresses: principe@cimne.upc.edu (J. Principe), ramon.codina@upc.edu (R. Codina).
}

approximations, is found as the solution a local problems driven by the residual through the Green function approach. The equivalence between the residual free bubble and the variational multiscale method was established in [6]. Other methods introduced to solve this problem are the Characteristic Galerkin method [20] and the Taylor Galerkin method [19]. A comparison of all these methods was performed in [12]. A recent review of stabilization techniques for the CDR equation can be found in [26].

Another problem identified in the Galerkin approximation of the CDR equation is the lack of stability when the reaction term is important, which manifests as numerical oscillations localized near boundary layers. The methods mentioned lead to a stable discrete formulation, but some of them (VMM) are much more accurate than others (GLS). The expression of the stabilization parameter needs to be modified to take reaction into account. An expression based on the satisfaction of the discrete maximum principle was proposed in [12]. If we denote the diffusion coefficient by $\varepsilon$, the norm of the advective velocity by $a$ and the reaction by $s$, this expression reads

$\tau=\left(\frac{c_{1} \varepsilon}{h^{2}}+\frac{c_{2} a}{h}+s\right)^{-1}$,

where $h$ is a characteristic element length and $c_{1}$ and $c_{2}$ are constants whose values, determined by numerical experiments, are 4 and 2, respectively. The expression proposed in [25] for the convection-diffusion case, based on the error analysis, was extended to the reactive case in [30], obtaining an expression that behaves asymptotically as (1), which means that the limits of the expression with respect to any of the equation coefficients and with respect to mesh size are the same. 
The dependence of the stabilization parameters with respect to the equation coefficients and the mesh size can be determined by the error analysis. However, as pointed out in [32], convergence proofs are performed using functional analysis inequalities that depend on unknown constants. This is sufficient as the error bounds are obtained up to a constant. Therefore, constants appearing in (1) cannot be determined by error analysis except in particular problems. At the same time, the analysis is performed under strong assumptions on the mesh, such as regularity of the elements or quasi-uniformity, and general definitions of the mesh size parameter $h$ are used (like the maximum or minimum element length for example). On the other hand, precise definitions of the constants and the mesh parameter $h$ are implemented in finite element codes, which are then used to solve application problems in meshes that are far from satisfying these constrains. The performance of the stabilized method presented in [50] (which is similar to the variational multiscale method in the context of the NavierStokes equations) when high aspect ratio elements are used was analyzed in [44] and the need for incorporating the stretching of the grid in the definition of the stabilization parameter was emphasized. Similar conclusions were obtained for linear elements in [31], where the performance of different stabilization methods using different definitions of the element length was analyzed.

An important effort in this direction is reported in [23] and references therein, where anisotropic error estimates are developed for the convection-diffusion equation using linear elements. Still some assumptions on the mesh are needed and the final error

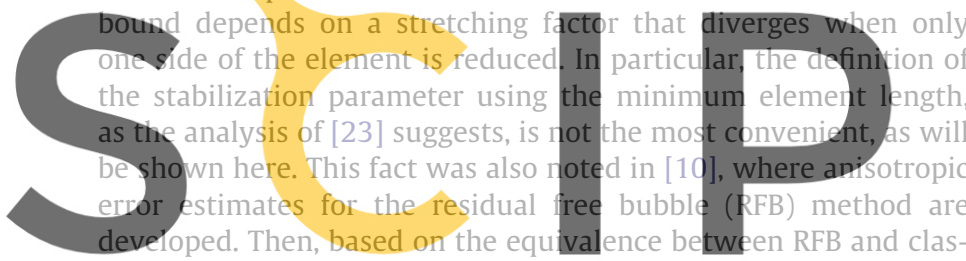
sical stabilization techniques, a new definition of the stabilization parameter is proposed on two-dimensional anisotropic triangula-

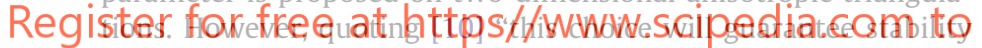
for any, not too unreasonably designed partition" meaning aspect ratios less than 5.

Another way in which the element length has been incorporated into the definition of the stabilization parameter is through the Jacobian of the isoparametric transformation, as in $[41,47]$. A completely different approach, based on the calculation of norm of the element matrices and vectors, is presented in [49]. We can also mention the finite calculus (FIC) method, based on expressing the equation of balance of fluxes in a domain of finite size, originally proposed in [45] and modified in [46] by the introduction of a nonlinear stabilization parameter. Another attempt to incorporate the anisotropy of the mesh into the definition of the stabilization parameters can be found in [4], in this case for the Stokes problem and based on taking $\tau$ a matrix rather than a scalar.

Although stabilization techniques have been extended to consider many different kinds of problems, a general definition of the stabilization parameters is still an open problem. In this work this definition of the stabilization parameters for scalar convection-diffusion equations is revisited and a new definition is proposed, which contains a precise definition of the element length and the values of the constants. Particular emphasis is put on the case of anisotropic mesh refinements. Even though our arguments are necessarily heuristic, we support them with the discussion of some particular cases, the numerical results and some facts drawn from convergence analysis.

The article is organized as follows. In Section 2 we state the problem to be solved including the discrete formulation, which is based on the variational multiscale method of $[37,39]$. In Section 3 the method to find an approximate solution of the fine scale problem is presented, the functional form of the stabilization parameter is defined and the final expression of the stabilization parameter is proposed. Section 4 presents a discussion of some particular cases illustrating the general approach and showing the problems that may appear with other definitions in contrast to the one proposed here. In Section 5 some comments on the standard error analysis of the method are made. As usual, convergence estimates depend on interpolation estimates which are difficult to obtain in the anisotropic case (see [10,2] and references therein). However, the analysis clearly shows that the stabilization parameter cannot be defined using the minimum element length. Numerical experiments illustrating the benefits of the definition proposed here are presented in Section 6 and final conclusions are drawn in Section 7.

\section{Problem statement}

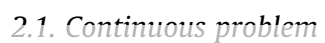

Let us consider a convection-diffusion-reaction problem, consisting of finding a function $u$ such that

\section{$\mathscr{L} u:=-\partial_{i}\left(\varepsilon_{i j} \partial_{j} u\right)+a_{i} \partial_{i} u+s u=f$ in $\Omega$ \\ $u=0$ on $\partial \Omega$}

Here, $\Omega \subset \mathbb{R}^{d}$ is an open domain ( $d=2,3$ is the number of space dimensions) and $\partial \Omega$ its boundary, $\varepsilon_{i j}$ is the constant (symmetric and positive definite) diffusion tensor, $a_{i}$ the solenoidal advection
velocity, $s \geqslant 0$ the constant reaction coefficient and $f$ a given inter-
nal force (the index sumnation convention over the plumber of
space dimensions is used here and in what follows). We restrict
ourselves to the case of positive reaction, which corresponds to
the exponential regime, and we refer to [36,33] for the case of neg-
ative reaction, which corresponds to the propagdtion regime.
As ustual, the space of functions whose power $(1 \leqslant p<\infty)$ is integrable in a domain $\omega$ is denoted by $L^{p}(\omega)$, and when $p=2$

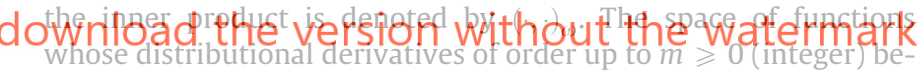
long to $L^{2}(\omega)$ is denoted by $H^{m}(\omega)$. The space $H_{0}^{1}(\omega)$ consists of functions in $H^{1}(\omega)$ vanishing on $\partial \omega$. The topological dual of $H_{0}^{1}(\omega)$ is denoted by $H^{-1}(\omega)$ and $\langle\cdot, \cdot\rangle_{\omega}$ is used to denote the duality pairing between them.

The problem can be written in a weak form as follows: given $f \in H^{-1}(\Omega)$, find $u \in V:=H_{0}^{1}(\Omega)$ such that

$B(u, v)=L(v) \quad \forall v \in V$,

where

$B(u, v)=\left(\partial_{i} v, \varepsilon_{i j} \partial_{j} u\right)_{\Omega}+\left(v, a_{i} \partial_{i} u\right)_{\Omega}+(v, s u)_{\Omega}$,

$L(v)=\langle v, f\rangle_{\Omega}$.

The discretization of the problem is based on a finite element partition of the domain, $\mathscr{P}_{h}=\{K\}$, of size $h>0$, which is a set of $n_{\text {el }}$ elements $K$ such that they cover the domain and their interiors are disjoint. Based on this partition, space $V$ is approximated by a finite dimensional space $V_{h}$ defined as

$V_{h}=\left\{\left.w \in V w \circ F^{-1}\right|_{K} \in P_{p}(\widehat{K}), 1 \leqslant p \leqslant \infty\right\}$,

where $P_{p}(\widehat{K})$ denotes the set of polynomials of degree at most $p$ (on each space variable if quadrilateral/hexahedral elements are used) and $F$ is the affine mapping from the reference element $\widehat{K}$ to the physical element $K$. The Galerkin discrete problem consists in finding $u_{h} \in V_{h}$ such that

$B\left(u_{h}, v_{h}\right)=L\left(v_{h}\right) \quad \forall v_{h} \in V_{h}$

This formulation is not stable if diffusive terms are small compared either to convective or reactive ones. 


\subsection{Two-scale decomposition}

Different stabilization techniques are used depending on the instability of the problem under consideration. A rather general method (that can be used in many cases) is the variational multiscale formulation. It is based on a decomposition of the unknown $u$ into a resolvable part $u_{h}$ and a subgrid scale part $\tilde{u}$ which cannot be captured by the finite element mesh, which corresponds to a decomposition of the space $V$ as

$V=V_{h} \oplus \widetilde{V}$.

The above decomposition, applied to the weak form of the problem, leads to

$$
\begin{aligned}
& B\left(u_{h}, v_{h}\right)+B\left(\tilde{u}, v_{h}\right)=L\left(v_{h}\right) \quad \forall v_{h} \in V_{h}, \\
& B\left(u_{h}, \tilde{v}\right)+B(\tilde{u}, \tilde{v})=L(\tilde{v}) \quad \forall \tilde{v} \in \widetilde{V} .
\end{aligned}
$$

The first equation is the equation for the resolvable scale $u_{h}$ and has two terms: the first one is the Galerkin contribution and the second one takes into account the influence of the subgrid scale on $u_{h}$. The second one is an equation for the subgrid scale contribution.

Let us introduce the following notation:

$\Omega^{h}=\cup_{K \in \mathscr{P}_{h}} K$ and $\Gamma^{h}=\cup_{K \in \mathscr{P}_{h}} \partial K$

and

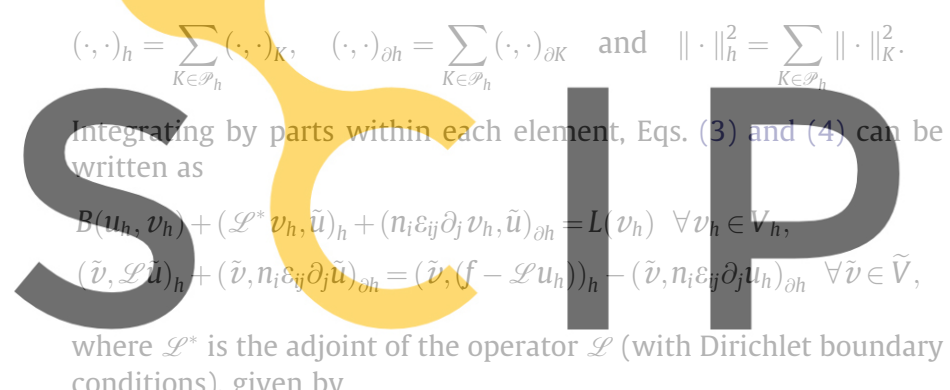

Register for fre at thattps//www.scipedia.com to d

As the normal fluxes of the exact solution are continuous across any suiface, it follows that

$\left(\tilde{v}, n_{i} \varepsilon_{i j} \partial_{j} u\right)_{\partial h}=\left(\tilde{v}, n_{i} \varepsilon_{i j} \partial_{j} \tilde{u}\right)_{\partial h}+\left(\tilde{v}, n_{i} \varepsilon_{i j} \partial_{j} u_{h}\right)_{\partial h}=0$

(note that $\tilde{v}=0$ on $\partial \Omega$ ). Then, the second equation is equivalent to: find $\tilde{u} \in \widetilde{V}$ such that

$\mathscr{L} \tilde{u}=f-\mathscr{L} u_{h}+\widetilde{v}^{\perp}$ in $\Omega^{h}$,

$\tilde{u}=u_{\text {ske }}$ on $\Gamma^{h}$,

where $u_{\text {ske }}$ is a function defined on the element boundaries and $\tilde{v}^{\perp}$ is any function in $\widetilde{V}^{\perp}$ (the orthogonal complement of $\widetilde{V}$ in the $L^{2}\left(\Omega^{h}\right)$ sense). The function $u_{\text {ske }}$ must be such that the normal fluxes of $u$ are continuous across interior element boundaries and defined as $u_{\text {ske }}=u-u_{h}$ on $\Gamma^{h}$. In turn, the function $\tilde{v}^{\perp}$ is responsible for guaranteeing that $\tilde{u} \in \tilde{V}$. A modeling step is necessary to solve the system, which means a choice of $u$ ske,$\tilde{v}^{\perp}$ and an approximate solution of (5).

Note that (5) is posed in $\Omega^{h}$, which consists of the union of the elements of the mesh. Therefore, any choice of $u_{\text {ske }}$ leads to $n_{\mathrm{el}}$ uncoupled problems posed on each element $K$. As a discrete approximation that gives exact nodal values would be optimal, one may ask the subscales to vanish at the nodes. In one dimensional problems, this gives homogeneous boundary conditions for problems (5), which are now decoupled and can be solved on each element. This has been done for the convection-diffusion and Helmholtz equations (see [39] and the references therein). In more than one space dimension the choice $u_{\text {ske }}=0$ is an approximation.
Another possibility has been explored in [17], where $u_{\text {ske }}$ is defined approximately imposing continuity of fluxes.

The approximated solution that will be constructed in the following section can be written as

$\left.\tilde{u}\right|_{K}=\left.\left.\mathscr{L}^{-1}\left[\left(f-\mathscr{L} u_{h}\right)+\tilde{v}^{\perp}\right]\right|_{K} \simeq \tau_{K}\left[\left(f-\mathscr{L} u_{h}\right)+\tilde{v}^{\perp}\right]\right|_{K}$.

This equation emphasizes that $\tau_{K}$ is an approximation to the (formal) inverse of the differential operator on each element $K$, a fact that will be used to construct an expression for it. Finally we have to impose $\tilde{u} \in \widetilde{V}$, or, equivalently, to define $\tilde{v}^{\perp}$. The simplest choice is to take $\tilde{v}^{\perp}=0$ which is called in [14] the Algebraic Subgrid-Scale formulation (ASGS). Another possibility is to take $\widetilde{V}$ as the orthogonal complement of $V_{h}$ in the $L^{2}\left(\Omega^{h}\right)$ sense, which requires the use of an $L^{2}\left(\Omega^{h}\right)$ projection with element-by-element weights $\tau_{K}$ [15]. However, as the $L^{2}\left(\Omega^{h}\right)$ projection is very convenient from a computational point of view, we neglect this variation. Therefore, the final approximation will be of the form

$\left.\tilde{u}\right|_{K}=\tau_{K} \Pi\left(f-\mathscr{L} u_{h}\right)$

where $\Pi=I$ ( $I$ is the identity in $V$ ) in the case of the ASGS method and $\Pi=P_{h}^{\perp}=I-P_{h}$ in the case of the OSS method, $P_{h}$ being the $L^{2}$ projection onto the finite element space. Let us emphasize that it is not our intention to discuss the choice of $\Pi$ (see [15] and the references therein) but the choice of $\tau_{K}$.

Neglecting boundary terms, the final stabilized discrete problem is: find $u_{h} \in V_{h}$ such that
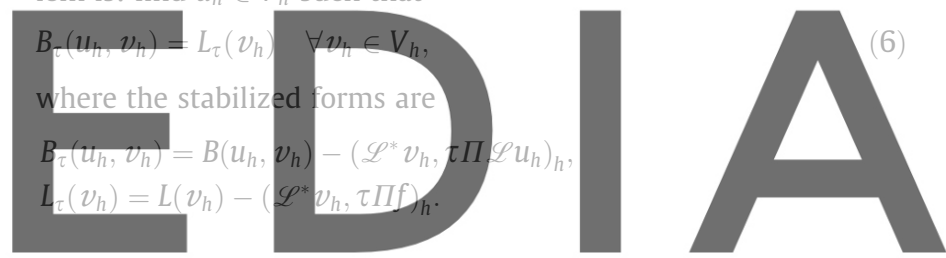

3. Approximate solution of the subscale equation download the version without the watermark
In this section an approximate solution of Eq. (5) is presented. This equation for the subscale can be thought as an equation for the error and it is the equation used for the derivation of a posteriori error estimators [1], a fact already noted in [39]. In fact, it is used as an error estimator in [22,34,35]. Two approaches are typical in a posteriori error estimation: an explicit expression for the error based on the residuals (derived from this equation) or the numerical solution of this equation (the so-called implicit methods) [1]. In this case the first approach is followed, because the problem is actually solved a priori and the relation between the subscale (the error) and the residual is used to stabilize the finite element problem.

The approximate solution is based on two properties that will be analyzed in Sections 3.1 and 3.2. The first is how the subgrid scale depends on the element size and will be determined by transforming the fine scale equation to the reference domain. The isoparametric transformation to the reference domain as a tool to define the stabilization parameters was used first in $[41,47]$, but only for implementation purposes and not related to the fine scale equation in the variational multiscale context that was developed later on in [39]. The second property is how the subgrid scale depends on the coefficients of the equation and will be determined by a heuristic argument already presented in [14] that will be revisited and extended. In this section we consider $\tilde{v}^{\perp}=0(\Pi=I)$ as it does not affect the discussion and we will discuss the choice of $\Pi$ in Section 5.

These two properties determine the functional form of the stabilization parameters but not their final form, which depends on some choices described later on. Sections 3.3 and 3.4 describe 
two possibilities, an isotropic definition widely used in the literature and the new anisotropic definition we propose.

\subsection{Transformation to the reference domain}

We are interested in anisotropic finite element meshes, and therefore on distorted elements. This is why instead of directly solving

$$
\begin{aligned}
& \mathscr{L} \tilde{u}=f-\mathscr{L} u_{h}:=r \text { in } K, \\
& \widetilde{u}=0 \text { on } \partial K
\end{aligned}
$$

on each element $K$, we will transform this equation to the reference domain. The isoparametric transformation is defined by the mapping $\mathbf{x}=F(\boldsymbol{\xi}$ ), relating the element $K$ (with coordinates $\mathbf{x}$ ) to the reference element $\widehat{K}$ (with coordinates $\xi$ ), and whose Jacobian $(J)$ verifies

$J_{k l}=\frac{\partial x_{l}}{\partial \xi_{k}}, \quad J_{k l}^{-t}=\frac{\partial \xi_{k}}{\partial x_{l}}$

Therefore, we can write the fine scale problem as

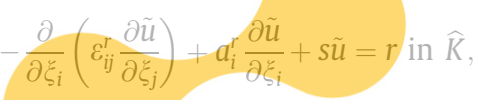

where the modified velocity and diffusion coefficients are defined by

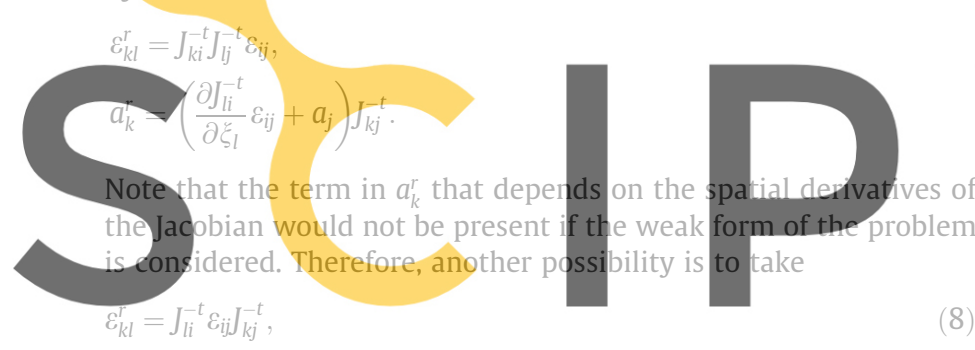

Register for free at https//www.scipedia.com to

\subsection{A Fourier analysis of the subscale problem}

As in [14], let us consider the Fourier transform of a function $v$ defined in $\widehat{K}$ as

$\hat{v}(\mathbf{k})=\int_{\widehat{K}} \mathrm{e}^{-i k \cdot \xi} v(\xi) \mathrm{d} \xi$,

where $i=\sqrt{-1}$ and $\mathbf{k}$ is the vector wave number. If $\mathbf{n}$ denotes the normal to the element $\widehat{K}$ we have that

$\frac{\widehat{\partial v}}{\partial \xi_{j}}(\mathbf{k})=\mathrm{i} k_{j} \hat{v}(\mathbf{k})+\int_{\widehat{\partial K}} n_{j} \mathrm{e}^{-\mathrm{i} k \cdot \xi} v \mathrm{~d} \xi$.

When this transform is applied to functions that vanish on the element boundary, the second term on the right-hand side vanishes and we have

$\frac{\partial v}{\partial \xi_{j}}(\mathbf{k})=\mathrm{i} k_{j} \widehat{v}(\mathbf{k})$.

Transforming Eq. (7) we arrive to

$\mathscr{T}^{-1}(\mathbf{k}) \hat{\tilde{u}}=\hat{r}$,

where

$\mathscr{T}^{-1}(\mathbf{k}):=\left(k_{i} k_{j} \varepsilon_{i j}^{r}+s+\mathrm{i} k_{j} a_{j}^{r}\right)$.

Note that $\mathscr{T}^{-1}(\mathbf{k})$ is the Fourier transform of the differential operator $\mathscr{L}$. It is well known that numerical instabilities appear when the convective or reactive terms are important compared to the diffu- sive one. In the Fourier context, the relative importance of these terms can be seen defining the directional Péclet and Damköhler numbers,

$\mathrm{P}_{\boldsymbol{k}}=\frac{k_{j} a_{j}^{r}}{k_{i} k_{j} \varepsilon_{i j}^{r}}, \quad \mathrm{D}_{\boldsymbol{k}}=\frac{s}{k_{i} k_{j} \varepsilon_{i j}^{r}}$

and writing

$\mathscr{T}^{-1}(\mathbf{k})=k_{i} k_{j} \varepsilon_{i j}^{r}\left(1+\mathrm{D}_{\boldsymbol{k}}+\mathrm{iP}_{\boldsymbol{k}}\right)$

Using the inverse Fourier transform the subgrid scale can be written as

$\tilde{u}(\boldsymbol{\eta})=\int_{\mathbb{R}^{\mathrm{d}}} \mathrm{e}^{\mathrm{i} \boldsymbol{k} \cdot \boldsymbol{\eta}} \hat{\tilde{u}}(\mathbf{k}) \mathrm{d} \mathbf{k}$

It is to be noted that the exact solution to the problem will depend on the element domain and the integration on the wave number space will be replaced by a sum over the values of $\mathbf{k}$ that make boundary conditions to be satisfied. In the above expression we can identify the Fourier representation of the Green function of the subscale problem [37], given by

$\tilde{u}(\boldsymbol{\eta})=\int_{\widehat{K}} G(\xi, \eta) r(\xi) \mathrm{d} \xi$,

where

$G(\xi, \eta)=\int_{n d}\left(k_{i} k_{j} \varepsilon_{i j}^{r}+s+i k_{j} a_{j}^{r}\right)^{-1} e^{-i k \cdot(\xi-\eta)} \mathrm{d} \mathbf{k}$

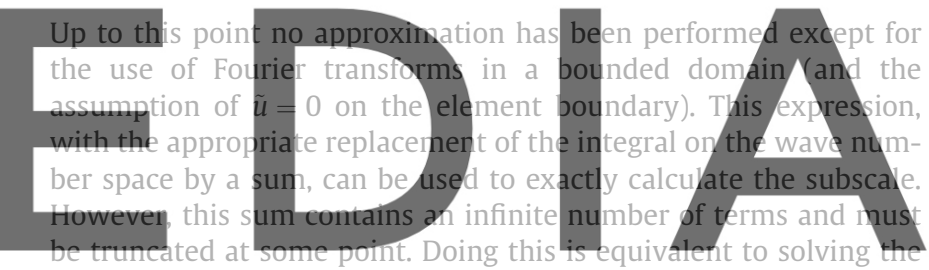

fine scale problem with a discrete formulation, which has already

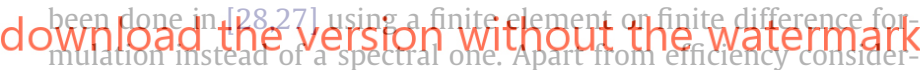
ations such approach has a conceptual problem: the fine scale problem will suffer the same numerical instability as the problem defined in $V_{h}$. Although in this problem the mentioned instability will not manifest when the submesh is fine enough, this is not the case when other problems (i.e. Stokes) are solved and the extension of the method will be difficult. Our main concern here is to find an approximation of (12).

It is well known $[12,37]$ that the use of a stabilization parameter $\tau$ corresponds to the approximation

$G(\boldsymbol{\xi}, \boldsymbol{\eta})=\tau \delta(\boldsymbol{\xi}, \boldsymbol{\eta})$,

where $\delta$ denotes the Dirac distribution. From expression (12) it is clear that this corresponds to the approximation

$$
\begin{aligned}
G(\boldsymbol{\xi}, \boldsymbol{\eta}) & \approx\left|\left(k_{i}^{0} k_{j}^{0} \varepsilon_{i j}^{r}+s+\mathrm{i} k_{j}^{0} a_{j}^{r}\right)^{-1}\right| \int_{\mathbb{R}^{\mathrm{d}}} e^{-\mathrm{i} \boldsymbol{k} \cdot(\boldsymbol{\xi}-\boldsymbol{\eta})} d \mathbf{k} \\
& =\left[\left(k_{i}^{0} k_{j}^{0} \varepsilon_{i j}^{r}+s\right)^{2}+\left(k_{j}^{0} a_{j}^{r}\right)^{2}\right]^{-1 / 2} \delta(\boldsymbol{\xi}-\boldsymbol{\eta})
\end{aligned}
$$

for some $\mathbf{k}^{0}$ to be defined, and then

$\tau=\left[\left(k_{i}^{0} k_{j}^{0} \varepsilon_{i j}^{r}+s\right)^{2}+\left(k_{j}^{0} a_{j}^{r}\right)^{2}\right]^{-1 / 2}$.

A justification for this approximation was presented in [14] where, using the mean value theorem, it was shown that if we approximate the subscale as $\widetilde{u}^{\text {ap }}=\tau r$ there exists $\mathbf{k}^{0}$ such that, defining $\tau$ as $\tau=\left|\mathscr{T}\left(\mathbf{k}^{0}\right)\right|$ the exact and the approximated subscales have the same norm, that is

$\|\tilde{u}\|_{L^{2}(\widehat{K})}^{2}=\left\|\tilde{u}^{\mathrm{ap}}\right\|_{L^{2}(\widehat{K})}^{2}$. 
Having established the functional form of the stabilization parameters let us finally consider the definition of $\mathbf{k}^{0}$, whose superscript will be omitted in the following. Taking into account (8) and (9) it is clear that a choice of $\mathbf{k}^{0}$ in (13) is equivalent to a choice the algorithmic constants $c_{1}$ and $c_{2}$ and a definition of the element length $h$ in (1). Moreover,

$k_{i} k_{j} \varepsilon_{i j}^{r}=\|\mathbf{k}\|^{2}\left(\frac{k_{i} k_{j}}{\|\mathbf{k}\|^{2}} \varepsilon_{i j}^{r}\right), \quad k_{j}^{0} a_{j}^{r}=\|\mathbf{k}\|\left(\frac{k_{i}}{\|\mathbf{k}\|} a_{j}^{r}\right)$

and the second terms in these products depend only on the direction of $\mathbf{k}$. Therefore, a definition of the direction of $\mathbf{k}$ implies a definition of $h$ in (1), and a definition of the norm of $\mathbf{k}$ implies a definition of the constants $c_{1}$ and $c_{2}$ in (1). Likewise, it immediately follows that $c_{1}=c_{2}^{2}$.

In order to determine $\|\mathbf{k}\|$ we can rely on numerical experiments or, as shown in the next section, on recovering optimal results in one dimensional problems. It will be shown in Section 4 that optimality in one dimensional problems results, for linear elements, in $\|\mathbf{k}\|=2 h_{\text {nat }}^{-1}$, where $h_{\text {nat }}$ is the size of the reference domain ( 2 for quads/bricks and 1 for triangles/tetrahedra).

The direction of $\mathbf{k}$ can be ignored performing invariant approximations, as shown in the following subsection, and when this is done the anisotropy of the problem is not considered. Another possibility is to introduce an intrinsic definition of the direction of $\mathbf{k}$, that is, a definition based on the coefficients of the equation and the mesh information available in the Jacobians of the isoparametric mapping. A proposal in this direction, taking into account the anisotropy of the problem, is presented below.
3.3. The isotropic case: recovering the usud expression
The definition of the stabilization paramete given in (13) is
invariant under transformations of the reference system. It is pos-
sible to preserve this invariance and to ignore the direction of $\mathbf{k}$, performing approximations of the products $k_{j} a_{j}^{r}$ and $k_{i} k_{j} \varepsilon_{i j}^{r}$ in terms

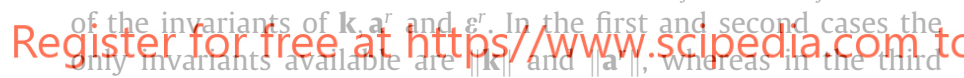
we have three possibilities. Considering the second invariant of $\boldsymbol{\varepsilon}^{r}\left(\sqrt{\varepsilon_{i j}^{r} \varepsilon_{i j}^{r}}\right)$ we can perform the approximations

$k_{j} a_{j}^{r} \simeq\|\mathbf{k}\|\left\|\mathbf{a}^{r}\right\|$

and

$k_{i} k_{j} \varepsilon_{i j}^{r} \simeq\|\mathbf{k}\|^{2} \sqrt{\varepsilon_{i j}^{r} \varepsilon_{i j}^{r}}$

Defining $c_{2}=\|\mathbf{k}\|$ and $c_{1}=c_{2}^{2}$ we arrive to the expression

$\tau=\left[\left(c_{1} \sqrt{\varepsilon_{i j}^{r} \varepsilon_{i j}^{r}}+s\right)^{2}+c_{2}\left\|\mathbf{a}^{r}\right\|^{2}\right]^{-1 / 2}$.

When $s=0$ and the diffusion coefficient $\varepsilon_{i j}$ is isotropic (given by $\varepsilon_{i j}=\varepsilon \delta_{i j}$ ) we have

$\varepsilon_{i j}^{r} \varepsilon_{i j}^{r}=\varepsilon^{2} J_{i j}^{-t} J_{i j}^{-t}=\varepsilon^{2} \frac{\partial \xi_{i}}{\partial x_{j}} \frac{\partial \xi_{i}}{\partial x_{j}}$

and

$\left\|\mathbf{a}^{r}\right\|^{2}=a_{i} J_{k i}^{-t} a_{j} J_{k j}^{-t}=a_{i} \frac{\partial \xi_{k}}{\partial x_{i}} \frac{\partial \xi_{k}}{\partial x_{j}} a_{j}$.

In this case

$\tau=\left(c_{1} \varepsilon^{2} g_{i j} g_{i j}+c_{2} a_{i} g_{i j} a_{j}\right)^{-1 / 2}$,

where

$g_{i j}=\frac{\partial \xi_{k}}{\partial x_{i}} \frac{\partial \xi_{k}}{\partial x_{j}}$ is the metric tensor related to the isoparametric mapping. The stabilization parameter defined by (17) was proposed first in [47] in the context of compressible flow equations and has been used in several applications $[48,51,9]$. The approximations (14) and (15) do not take into account the angle between the equation coefficients and the vector $\mathbf{k}$. It is due to these approximations, based on invariant quantities only, that the information on the anisotropy of the mesh is lost. Another possibility is analyzed in the following subsection.

\subsection{The general case: proposed expression}

In order to consider the anisotropy of the problem, an intrinsic definition of the direction of $\mathbf{k}$ in terms of the data must be given. Our proposal is based on the following heuristic argument. As it is well known, numerical instabilities may occur when the convective or reactive terms are important and they are larger when these numbers increase. In the case of variable coefficients or mesh sizes numerical instabilities are important where these numbers are larger. In the case of anisotropic problems we propose to find the direction in which these numbers are bigger, that is, the direction of maximum instability of the problem. This is possible thanks to the definition of the directional dimensionless Pé clet and Damköhler numbers (10). Therefore, as a definition of the direction of $\mathbf{k}$, we propose the direction in which some combination of $D_{k}$ and $\mathrm{P}_{\boldsymbol{k}}$ is maximum. From (13) we have

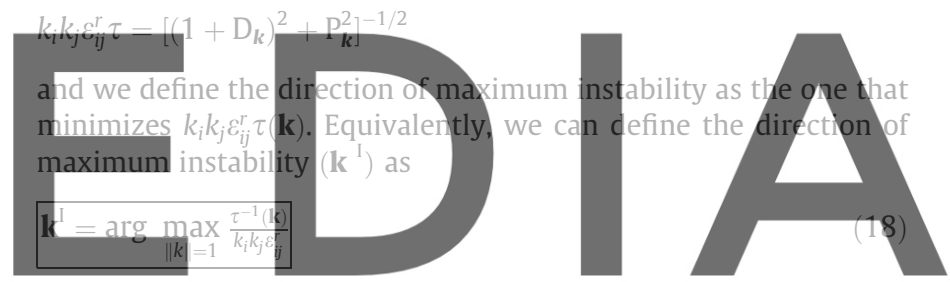

and the stabilization parameter we propose is given by $\tau\left(\mathbf{k}^{\mathrm{I}}\right)$, that is,

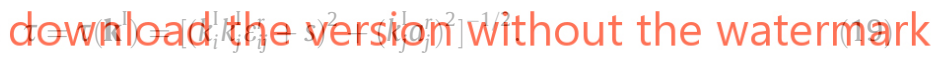

In the following section we present some heuristic arguments supporting this proposal, and in Section 6 its benefits are illustrated in some numerical experiments. We will also show how other choices of $\mathbf{k}$ lead to different methods that can be found in the literature.

\section{Discussion}

In this section several aspects of the proposed approach in some particular cases are discussed. We first show how the values of the algorithmic constants can be found analyzing one dimensional problems. Then we illustrate how the definition of the element length in terms of the direction of maximum instability of the problem works in a simple setting. After that, an analytic expression of $\mathbf{k}^{\mathrm{I}}$ is presented for some particular problems, together with a simple algorithm for the general case. We conclude presenting alternative definitions considered in the literature and simple cases in which they fail or are not optimal.

\subsection{Algorithmic constants for $1 D$ optimal results}

Let us consider the problem in one space dimension without reaction. In this case the stabilization parameter presented above (see (13)) is given by

$\tau=\left[\left(\frac{h_{\mathrm{nat}}^{2} k^{2} \varepsilon}{h^{2}}\right)^{2}+\left(\frac{h_{\mathrm{nat}} k a}{h}\right)^{2}\right]^{-1 / 2}$ 
and using the Péclet number defined as

$\mathrm{P}=\frac{a h}{2 \varepsilon}$

it can be written in dimensionless form as

$\alpha=\frac{2 a \tau}{h}=\left[\left(\frac{h_{\mathrm{nat}}^{2} k^{2}}{4 \mathrm{P}^{2}}\right)^{2}+\left(\frac{h_{\mathrm{nat}} k}{2}\right)^{2}\right]^{-1 / 2}$.

The advective limit of this expression is

$\lim _{\mathrm{p} \rightarrow \infty} \alpha=2 h_{\mathrm{nat}}^{-1} k^{-1}$.

The analytic solution to the problem can be used to obtain the function $\alpha^{\text {opt }}$ that guarantees exact nodal values which, in the case of linear elements, is given by

$\alpha^{\mathrm{opt}}=\operatorname{coth}(\mathrm{P})-\frac{1}{\mathrm{D}}$

The advective limit of $\alpha^{\mathrm{opt}}$ is 1 and therefore we conclude that

$k=2 h_{\text {nat }}^{-1}$

must be taken. Both expressions, $\alpha$ and $\alpha^{\text {opt }}$, are compared in Fig. 1

for this choice of $k$.

Note that the final expression for the stabilization parameter does not depend on the reference domain, as expected. In the case of quadratic elements it is not possible to obtain exact nodal values

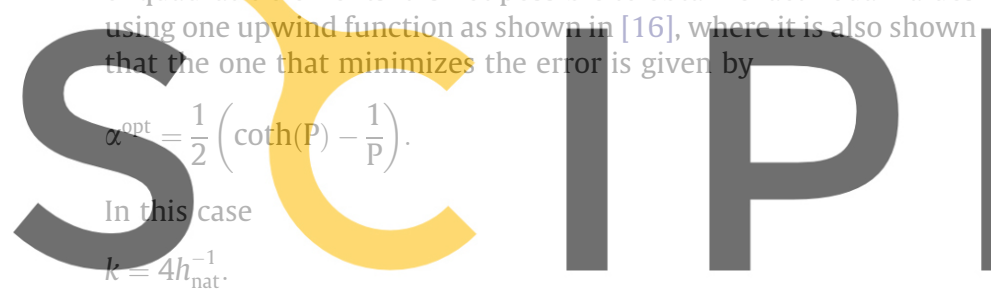

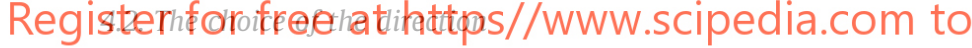

In order to get an insight of how the direction of $\mathbf{k}$ should be taken, let us consider two directions $\mathbf{k}_{1}=(1,0)$ and $\mathbf{k}_{2}=(0,1)$, and compare the stabilization parameter obtained using each of them in the following two examples, a convection-diffusion and a reaction-diffusion problem. These examples, and those presented in the following, are defined in the unit square, which is discretized using rectangular elements of sizes $h_{1}=1 / n_{1}$ and $h_{2}=1 / n_{2}, n_{1}$ and $n_{2}$ being the number of elements along each side of the domain. An isotropic diffusion $\varepsilon$ is considered. In this case, for a general velocity vector $\mathbf{a}=\left(a_{1}, a_{2}\right)^{t}$, the coefficients of the equation in the reference domain are

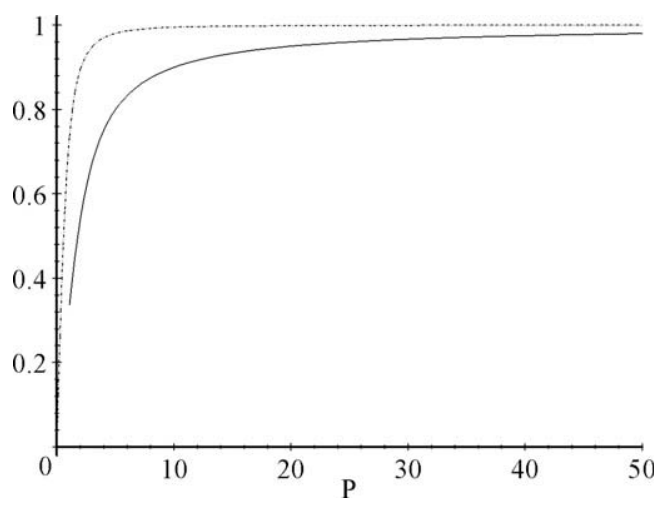

Fig. 1. Upwind functions for linear elements. Dotted line: $\alpha$. Solid line: $\alpha^{\mathrm{opt}}$. $\boldsymbol{\varepsilon}^{r}=\left[\begin{array}{cc}\frac{4}{h_{1}^{2}} \varepsilon & 0 \\ 0 & \frac{4}{h_{2}^{2}} \varepsilon\end{array}\right], \quad \mathbf{a}^{r}=\left[\begin{array}{c}\frac{2 a_{1}}{h_{1}} \\ \frac{2 a_{2}}{h_{2}}\end{array}\right]$

Remark 1. The subgrid problem solved in the reference domain will present an anisotropic diffusion when the mesh size is anisotropic. In other words, the anisotropy of the mesh gives rise to a subgrid scale problem with anisotropic diffusion coefficient.

Example 1. Let us consider a convection-diffusion problem with a velocity of the form $\mathbf{a}=(a, 0)^{t}$. In this case

$\tau\left(\mathbf{k}_{1}\right)=\left[\left(\frac{4 \varepsilon}{h_{1}^{2}}\right)^{2}+\left(\frac{2 a}{h_{1}}\right)^{2}\right]^{-1 / 2}, \quad \tau\left(\mathbf{k}_{2}\right)=\left(\frac{4 \varepsilon}{h_{2}^{2}}\right)^{-1}$.

If we consider a convection dominated problem in a uniform mesh of size $h_{1}=h_{2}=h$,

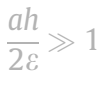

and

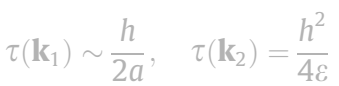

In this case

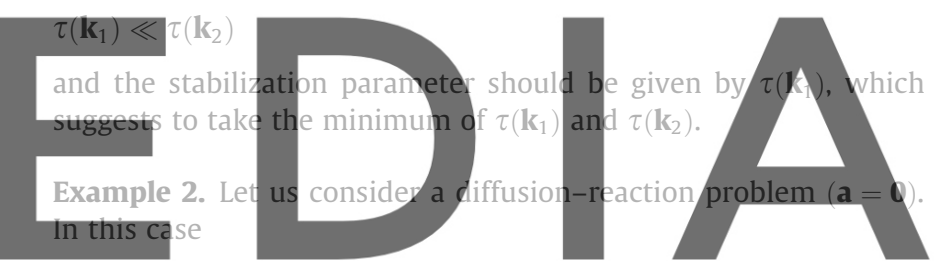

\section{$\tau\left(\mathbf{k}_{1}\right)=\left(\frac{4 \varepsilon}{2}+s\right)^{-1}, \tau\left(\mathbf{K}_{2}\right)=\left(\frac{4 \varepsilon}{2^{2}}+s\right.$}

download the version without the watermark

Let us consider a reaction dominated problem (small diffusion) and the two cases of anisotropic refinement. First if the mesh in direction 1 is very fine but is coarse in direction 2 then

$\frac{s h_{1}^{2}}{4 \varepsilon} \ll 1$ and $\frac{s h_{2}^{2}}{2 \varepsilon} \gg 1$

and we have

$\tau\left(\mathbf{k}_{1}\right) \sim \frac{h_{1}^{2}}{4 \varepsilon}, \quad \tau\left(\mathbf{k}_{2}\right) \sim \frac{1}{s}$,

which implies

$\tau\left(\mathbf{k}_{1}\right) \ll \tau\left(\mathbf{k}_{2}\right)$.

In this case the stabilization parameter should be given by $\tau\left(\mathbf{k}_{2}\right)$. Second, if the mesh in the direction 1 is coarse but in the direction 2 is fine enough, we can find similarly

$\tau\left(\mathbf{k}_{2}\right) \ll \tau\left(\mathbf{k}_{1}\right)$.

In this case the stabilization parameter should be given by $\tau\left(\mathbf{k}_{1}\right)$. Therefore, in the case of a pure reactive problem we could consider the maximum of $\tau\left(\mathbf{k}_{1}\right)$ and $\tau\left(\mathbf{k}_{2}\right)$ or simply their sum. This is equivalent to consider the direction $\mathbf{k}$ of maximum element length or, in other words, the direction of minimum diffusivity, i.e. the direction $\mathbf{k}$ that makes $k_{i} k_{j} \varepsilon_{i j}^{r}$ minimum.

The situation is similar in the two examples and gives rise to the conclusion that motivated the proposal: $\mathbf{k}$ depends on the direction in which the instability of the problem appears. In these examples the way to determine which is the correct definition of the stabiliza- 
tion parameter was by determining the direction in which the instability appears and this was done by comparing the directional dimensionless numbers (10), which in this case reduce to

$\mathrm{P}_{\boldsymbol{k}_{1}}=\frac{a h_{1}}{2 \varepsilon}, \quad \mathrm{P}_{\boldsymbol{k}_{2}}=\frac{a h_{2}}{2 \varepsilon}, \quad \mathrm{D}_{\boldsymbol{k}_{1}}=\frac{s h_{1}^{2}}{4 \varepsilon}, \quad \mathrm{D}_{\boldsymbol{k}_{2}}=\frac{s h_{2}^{2}}{2 \varepsilon}$

and the one that is dominant defines the direction that needs to be considered. The proposed expression (18) automatically detects these situations.

\subsection{The computation of the direction}

Definition (18) implies the maximization of the function

$H(\mathbf{k})=\frac{\tau^{-1}(\mathbf{k})}{k_{i} k_{j} \varepsilon_{i j}^{r}}=\left[\left(1+\frac{s}{k_{i} k_{j} \varepsilon_{i j}^{r}}\right)^{2}+\left(\frac{k_{j} a_{j}^{r}}{k_{i} k_{j} \varepsilon_{i j}^{r}}\right)^{2}\right]^{1 / 2}$

but, as the square root is a monotone function, we may solve the equivalent problem of maximizing $H^{2}(\mathbf{k})$. This optimization problem will be approximately solved. After multiplying its gradient by $\left(k_{i} k_{j} \varepsilon_{i j}^{r}\right)^{3}$ we arrive to the equation

$$
-\left[\left(k_{j} a_{j}^{r}\right)^{2}+\left(k_{i} k_{j} \varepsilon_{i j}^{r}+s\right) s\right] \nabla_{k}\left(k_{i} k_{j} \varepsilon_{i j}^{r}\right)+\left(k_{i} k_{j} \varepsilon_{i j}^{r}\right)\left(k_{j} a_{j}^{r}\right) \nabla_{k}\left(k_{j} a_{j}^{r}\right)=0,
$$

where $\nabla_{k}$ is the gradient with respect to $\mathbf{k}$. As the minimization is performed under the restriction $\|\mathbf{k}\|=1$, in $2 \mathrm{D}$ we can take $\mathbf{k}=(\cos \theta, \sin \theta)$ and after a change of variables of the form
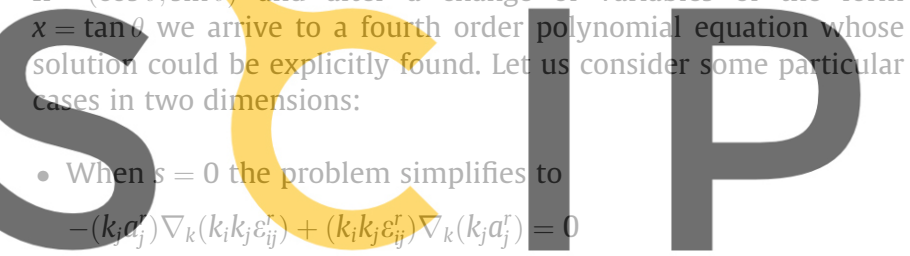

and after taking $\mathbf{k}=(\cos \theta, \sin \theta)$ we arrive to a third order polynoRegisterufor free at https//www.scipedia.com to $-a_{1}^{r}\left(\varepsilon_{12}^{r}+\varepsilon_{21}^{r}\right)+a_{2}^{r} \varepsilon_{11}^{r}+\left[a_{1}^{r} \varepsilon_{11}^{r}-2 a_{1}^{r} \varepsilon_{22}^{r}-2 a_{2}^{r}\left(\varepsilon_{12}^{r}+\varepsilon_{21}^{r}\right)\right] x$

$$
+\left[2 a_{2}^{r} \varepsilon_{11}^{r}-a_{2}^{r} \varepsilon_{22}^{r}\right] x^{2}+\left[a_{2}^{r}\left(\varepsilon_{12}^{r}+\varepsilon_{21}^{r}\right)-a_{1}^{r} \varepsilon_{22}^{r}\right] x^{3}=0,
$$

where $x=\tan \theta$. If we further assume an isotropic diffusion $\varepsilon$ and velocity $\mathbf{a}=(a, 0)^{t}$, this equation simplifies to

$\left[\varepsilon_{11}^{r}-2 \varepsilon_{22}^{r}\right] x-\varepsilon_{22}^{r} x^{3}=0$

and we have two possible solutions that can be found as illustrated in Fig. 2. (a) When the mesh is such that

$\varepsilon_{11}^{r}<2 \varepsilon_{22}^{r} \Longleftrightarrow \frac{4 \varepsilon}{h_{1}^{2}}<2 \frac{4 \varepsilon}{h_{2}^{2}} \Longleftrightarrow h_{2}^{2}<2 h_{1}^{2}$

or, equivalently

$\mathrm{A}:=\frac{h_{2}}{h_{1}}<\sqrt{2}$

we have the solution $\theta=0$.

(b) When the mesh is such that

$\varepsilon_{11}^{r}>2 \varepsilon_{22}^{r} \Longleftrightarrow \frac{4 \varepsilon}{h_{1}^{2}}>2 \frac{4 \varepsilon}{h_{2}^{2}} \Longleftrightarrow h_{2}^{2}>2 h_{1}^{2}$

or, equivalently

$\mathrm{A}=\frac{h_{2}}{h_{1}}>\sqrt{2}$

we have the solution

$\tan ^{2} \theta=\frac{\varepsilon_{11}^{r}-2 \varepsilon_{22}^{r}}{\varepsilon_{22}^{r}}=A^{2}-2$

In this case the stabilization parameter is given by
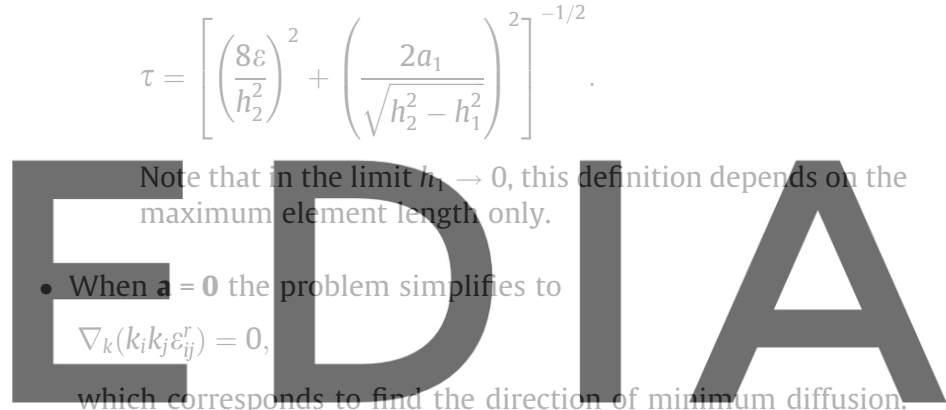

We can follow the same procedure used before to show that

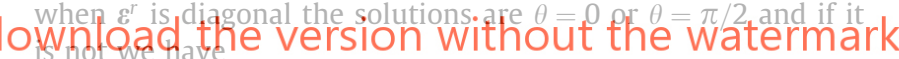

$$
\tan \theta=-\frac{\left(\varepsilon_{11}^{r}-\varepsilon_{22}^{r}\right)}{\left(\varepsilon_{12}^{r}+\varepsilon_{21}^{r}\right)} \pm\left(\frac{\left(\varepsilon_{11}^{r}-\varepsilon_{22}^{r}\right)^{2}}{\left(\varepsilon_{12}^{r}+\varepsilon_{21}^{r}\right)^{2}}+1\right)^{1 / 2}
$$

As these particular cases illustrate the maximum of $H(\mathbf{k})$ will occur somewhere between the direction of minimum diffusion and the direction of $\mathbf{a}^{r}$. Therefore, in practice, we find this maximum by bisection (golden section search), which usually converges in 3 or 4 steps.
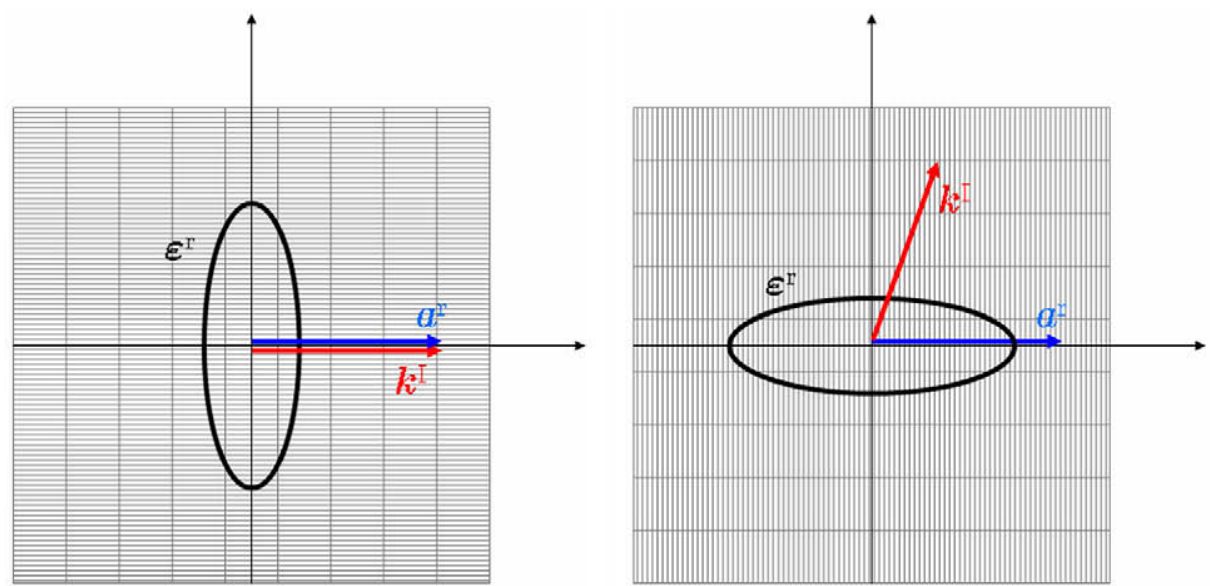

Fig. 2. The definition of the instability direction in cases (a) (left) and (b) (right). 


\subsection{Alternative definitions of $h$ (and their problems)}

Natural candidates for the definition of the element length have been widely used in the literature: the maximum element length $\left(h_{\max }\right)$, the minimum element length $\left(h_{\min }\right)$ and the streamline element length $\left(h_{a}\right)$. The definition of the element length in the direction of the flow was considered in [16,18] and in [44].

Let us first mention that these definitions can be obtained by a suitable choice of the vector $\mathbf{k}$, at least in the case of an isotropic diffusion coefficient $\varepsilon$, which illustrates how a definition of the direction of $\mathbf{k}$ implies a definition of the element length used. The maximum and minimum element lengths correspond to the minimum and maximum of the diffusion coefficient of the subscale problem transformed to the reference domain (see Remark 1). For linear elements they can be written as

$\frac{h_{\mathrm{nat}}^{2} \varepsilon}{h_{\mathrm{min}}^{2}}=\max _{\|\mathbf{k}\|=1} k_{i} k_{j} \varepsilon_{i j}^{r}, \quad \frac{h_{\mathrm{nat}}^{2} \varepsilon}{h_{\max }^{2}}=\min _{\|k\|=1} k_{i} k_{j} \varepsilon_{i j}^{r}$

In turn, the streamline element length $\left(h_{a}\right)$ naturally appears when we consider, for linear elements (otherwise the modulus of $\mathbf{k}$ should be changed as explained above), the choice
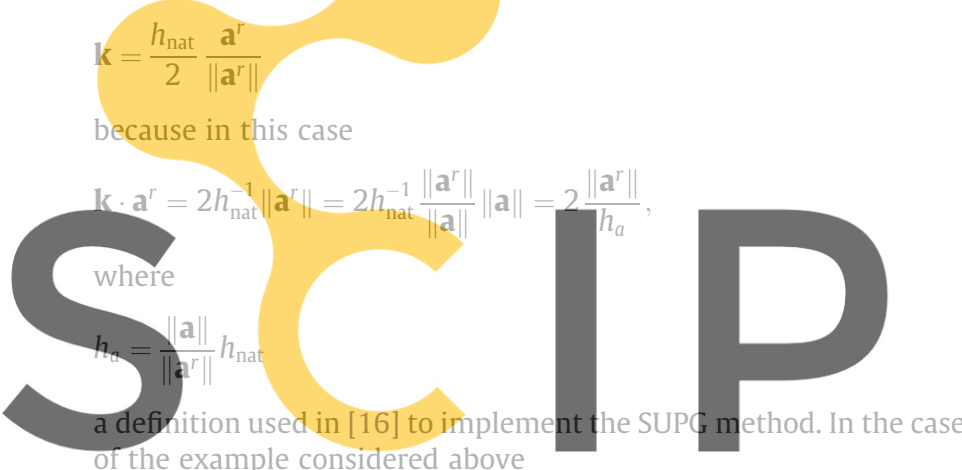

of the example considered above

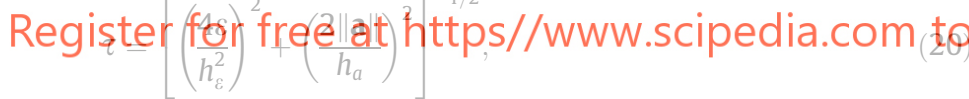

where

$h_{\varepsilon}^{2}=\left(\frac{a_{1}^{2}}{h_{1}^{2}}+\frac{a_{2}^{2}}{h_{2}^{2}}\right)\left(\frac{a_{1}^{2}}{h_{1}^{4}}+\frac{a_{2}^{2}}{h_{2}^{4}}\right)^{-1}$,

$h_{a}=\left(a_{1}^{2}+a_{2}^{2}\right)^{1 / 2}\left(\frac{a_{1}^{2}}{h_{1}^{2}}+\frac{a_{2}^{2}}{h_{2}^{2}}\right)^{-1 / 2}$.

Note that if $h_{1} \rightarrow 0$ and $h_{2}$ is fixed, $h_{\varepsilon} \rightarrow h_{1}$ and $h_{a} \rightarrow h_{1}$.

We can group the definitions of the stabilization parameter considered so far (those based on $h_{\max }, h_{\min }, h_{a}$ and (19)) according to their behavior in the anisotropic limit: (19) behaves as the definition using the maximum element length, as shown in the previous subsection, and the definition using the streamline element length behaves as the definition using the minimum element length, as shown before (except when the velocity is exactly zero in this direction).

Let us now present some examples showing that, under highly anisotropic refinement, the first group is not optimal in some cases but always results in a stable formulation, whereas the second one is more accurate than the first one in some cases but also unstable in others. Numerical results of these examples will be presented in Section 6.

Example 3. Consider a convection-diffusion problem with a velocity of the form $\mathbf{a}=(a, 0)^{t}$ and a mesh in which $h_{1} \rightarrow 0$, whereas $h_{2}$ is fixed (correct refinement). Defining the stabilization parameter using the minimum element length or the streamline element length, optimal results will be obtained whereas using maximum element length or (19) results will be overdiffusive.

Example 4. Consider a convection-diffusion problem with a velocity of the form $\mathbf{a}=(a, 0)^{t}$ and a mesh in which $h_{1}$ is fixed whereas $h_{2} \rightarrow 0$ (incorrect refinement). If we define the stabilization parameter using the minimum element length, $\tau \rightarrow 0$ and Galerkin instabilities will appear. Then, using the minimum element length results in an unstable method.

Example 5. Consider a convection-diffusion problem with a velocity of the form $\mathbf{a}=(a, a)^{t}$. If we define the stabilization parameter using (20) and $h_{1} \rightarrow 0$, then $h_{a} \rightarrow h_{1}$ and $h_{\varepsilon} \rightarrow h_{1}$ and again $\tau \rightarrow 0$, facing the same situation as in the previous example.

\section{Comments on the error analysis}

In this section we outline a standard error analysis of the method in the case of $\varepsilon_{i j}=\varepsilon \delta_{i j}$ and, in a particular situation where interpolation estimates are available, we show that using the minimum element length the convergence bound diverges under anisotropic refinement. We also explain why the method obtained using the maximum element length or (19) is not optimal in situations like those of Example 3 and we further show that in these cases the use of the orthogonal subgrid scales corrects the problem, giving optimal results.

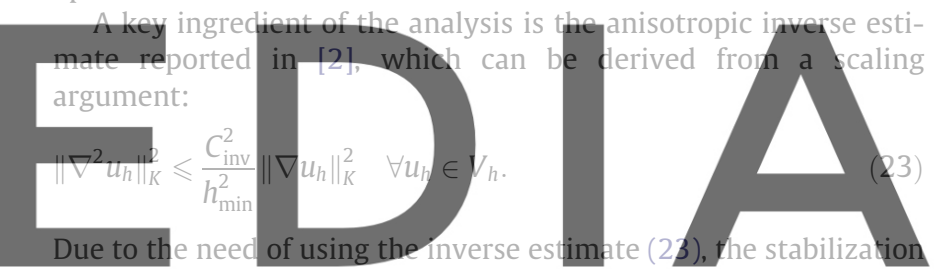

parameter must satisfy the following condition

\section{downløat the version without the watermark}

In the case of linear elements $C_{\text {inv }}=0$, and the above condition is automatically satisfied by (19) and (1). If higher order elements are used, only taking $h_{\min }$ in (1) will satisfy it. In this case, the direction of instability used in (19) should be modified to take this condition into account. In any case we stress that bound (23) might not be sharp and therefore (24), which is a sufficient condition for the analysis, might not be necessary. An estimation of the constant $C_{\text {inv }}$ can be found in [32].

The norm we will use for the analysis is defined by

$\left\|u_{h}\right\|_{\tau}^{2}=\varepsilon\left\|\nabla u_{h}\right\|_{\Omega}^{2}+\left\|\tilde{s}^{1 / 2} u_{h}\right\|_{h}^{2}+\left\|\tau^{1 / 2} \mathbf{a} \cdot \nabla u_{h}\right\|_{h}^{2}$,

where $\tilde{s}=s(1-\tau s)$ in the ASGS method and $\tilde{s}=s$ in the OSS method.

Lemma 1 (stability). Assume that the stabilization parameter satisfies condition (24). Then, there exists a constant $C>0$ such that

$\inf _{u_{h} \in V_{h}} \sup _{v_{h} \in V_{h}} \frac{B_{\tau}\left(u_{h}, v_{h}\right)}{\left\|u_{h}\right\|_{\tau}\left\|v_{h}\right\|_{\tau}} \geqslant C$

Proof. The proof is obtained following arguments similar to those used in [15] which are only sketched here. Taking $v_{h}=u_{h}$ in (6), considering the skew symmetry of the convective term and using the inverse estimate (23), condition (24) and the inequality $2 x y \leqslant \alpha^{-1} x^{2}+\alpha y^{2}$ permits to bound $\left\|\tau^{1 / 2} \Pi\left(\mathbf{a} \cdot \nabla u_{h}\right)\right\|_{h}^{2}$. In the case of $\Pi=I$ (ASGS method) this automatically gives

$B_{\tau}\left(u_{h}, u_{h}\right) \geqslant C\left\|u_{h}\right\|_{\tau}^{2}$ 
which implies the result. In the case of $\Pi=P_{h}^{\perp}=I-P_{h}$ (OSS method) taking $v_{h}=\tau P_{h}\left(\mathbf{a} \cdot \nabla u_{h}\right)$ and using the inverse estimate (23), condition (24) and the inequality $2 x y \leqslant \alpha^{-1} x^{2}+\alpha y^{2}$ permits to bound $\left\|\tau^{1 / 2} P_{h}\left(\mathbf{a} \cdot \nabla u_{h}\right)\right\|_{h}^{2}$. Given $u_{h}$ it can be shown that

$B_{\tau}\left(u_{h}, v_{h}\right) \geqslant C\left\|u_{h}\right\|_{\tau}\left\|v_{h}\right\|_{\tau}$,

where $v_{h}=u_{h}+\alpha \tau P_{h}\left(\mathbf{a} \cdot \nabla u_{h}\right)$ for a proper choice of $\alpha$. We refer the reader to [15] for the details.

Let us now consider $\hat{u}_{h}$ an interpolant of the solution of the continuous problem $u$ and define the interpolation error $\eta=u-\hat{u}_{h}$. We will present now a bound of $B_{\tau}\left(\eta, v_{h}\right)$ in terms of a function of the interpolation error $E(\eta)$ defined by

$$
\begin{aligned}
E(\eta)= & \varepsilon^{1 / 2}\|\nabla \eta\|_{\Omega}+\left\|\tau^{1 / 2} \Pi(\mathbf{a} \cdot \nabla \eta)\right\|_{h}+\left\|(s-\sigma)^{1 / 2} \eta\right\|_{h} \\
& +\left\|\tilde{\boldsymbol{s}}^{1 / 2} \Pi \eta\right\|_{h}+\varepsilon\left\|\tau^{1 / 2} \Pi\left(\nabla^{2} \eta\right)\right\|_{h}+\left\|\left(\tau^{-1}-\sigma\right)^{1 / 2} \eta\right\|_{h} \\
& +\left\|\frac{\tau \sigma}{\tilde{s}^{1 / 2}} \mathbf{a} \cdot \nabla \eta\right\|_{h},
\end{aligned}
$$

where $\sigma=s$ for the ASGS method whereas $\sigma=0$ for the OSS method. In turn, this function can be bounded relying on some results from interpolation theory, as discussed below.

Lemma 2. Assume that the stabilization parameter satisfies condition (24). Then

\section{$\|\eta\|_{\tau} \leqslant C E(\eta)$}

and

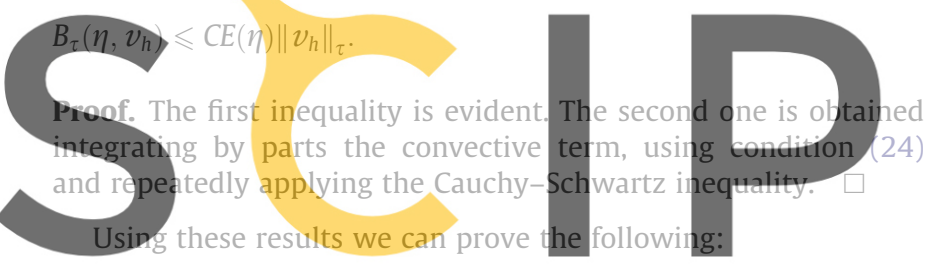

Theorem 1. Assume that the stabilization parameter satisfies condi-

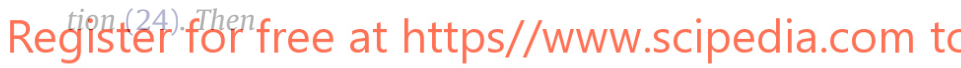
$\left\|u-u_{h}\right\|_{\tau} \leqslant C E(\eta)=C E\left(u-\hat{u}_{h}\right)$

where $E$ is the function defined in (25).

Remark 2. The only condition needed to prove convergence in the anisotropic case is (24). After satisfying this condition, there is still some freedom for the selection of the stabilization parameter (in the case of linear elements this condition is satisfied for any definition). Therefore, the difference between the definition (1) or (19) is the norm in which this convergence is proved and the form of the function $E(\eta)$ (which depends on $\tau$ ). In the isotropic case this estimate is optimal (see the discussion in [13] about the norm $\|\cdot\|_{\tau}$ ). In the anisotropic case, this optimality could be shown using appropriate interpolation estimates (see below). However, numerical experiments will show the convenience of choosing (19).

As mentioned, anisotropic interpolation estimates are difficult to obtain. Except those presented in [23], they are generally based on strong assumptions on the discretization, like the maximal angle condition [2]. We will use a result presented in [10] in the particular case of 2D bilinear elements:

Lemma 3. Assume that $K$ is a $2 D$ axiparallel element and $u \in H^{2}(\Omega)$ is interpolated by $\hat{u}_{h}$ using the first order tensor product operator defined in [10]. Then

$\|\eta\|_{K}^{2} \leqslant\left\|u-\hat{u}_{h}\right\|_{K}^{2} \leqslant C\left(h_{1}^{4}\left\|\frac{\partial^{2} u}{\partial x_{1}^{2}}\right\|_{K}^{2}+h_{2}^{4}\left\|\frac{\partial^{2} u}{\partial x_{2}^{2}}\right\|_{K}^{2}+h_{1}^{2} h_{2}^{2}\left\|\frac{\partial^{2} u}{\partial x_{2} \partial x_{1}}\right\|_{K}^{2}\right)$

and for $i=1,2$ $\left\|\partial_{i} \eta\right\|_{K}^{2} \leqslant\left\|\partial_{i}\left(u-\hat{u}_{h}\right)\right\|_{K}^{2} \leqslant C\left(h_{i}^{2}\left\|\frac{\partial^{2} u}{\partial x_{i}^{2}}\right\|_{K}^{2}+h_{i *}^{2}\left\|\frac{\partial^{2} u}{\partial x_{i *} \partial x_{i}}\right\|_{K}^{2}\right)$,

where $i_{*}=3-i$.

If we consider a convection-diffusion problem $(s=0)$, using that $\|\Pi\| \leqslant 1$ the error function can be bounded by

$$
\begin{aligned}
E(\eta) \leqslant & C\left(\varepsilon^{1 / 2}+\tau^{1 / 2}\left|a_{1}\right|_{K}\right)\left(h_{1}^{2}\left\|\frac{\partial^{2} u}{\partial x_{1}^{2}}\right\|_{K}^{2}+h_{2}^{2}\left\|\frac{\partial^{2} u}{\partial x_{2} \partial x_{1}}\right\|_{K}^{2}\right)^{1 / 2} \\
& +C\left(\varepsilon^{1 / 2}+\tau^{1 / 2}\left|a_{2}\right|_{K}\right)\left(h_{2}^{2}\left\|\frac{\partial^{2} u}{\partial x_{2}^{2}}\right\|_{K}^{2}+h_{1}^{2}\left\|\frac{\partial^{2} u}{\partial x_{2} \partial x_{1}}\right\|_{K}^{2}\right)^{1 / 2} \\
& +C \tau^{-1 / 2}\left(h_{1}^{4}\left\|\frac{\partial^{2} u}{\partial x_{1}^{2}}\right\|_{K}^{2}+h_{2}^{4}\left\|\frac{\partial^{2} u}{\partial x_{2}^{2}}\right\|_{K}^{2}+h_{1}^{2} h_{2}^{2}\left\|\frac{\partial^{2} u}{\partial x_{2} \partial x_{1}}\right\|_{K}^{2}\right)^{1 / 2} \\
& +4 \varepsilon \tau^{1 / 2}\left(\left\|\Pi \frac{\partial^{2} u}{\partial x_{1}^{2}}\right\|_{K}^{2}+\left\|\frac{\partial^{2} u}{\partial x_{2}^{2}}\right\|_{K}^{2}\right)^{1 / 2}
\end{aligned}
$$

Remark 3. Here it is clearly seen that, in the anisotropic limit, $\tau$ cannot be defined using the minimum element length or the streamline element length because the third term diverges as $h_{\mathrm{min}}^{-1 / 2}$ (except if the solution is one dimensional in the direction of $h_{\min }$ ).

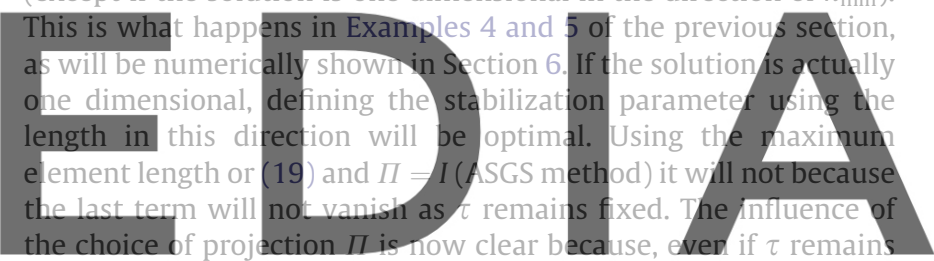
fixed, using $\Pi=P_{h}^{\perp}$ (OSS method) the last term vanishes (at a rate $h^{p+1}$ when polynomials of degree $p$ are used if $u$ is regular enough)

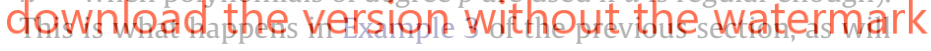
be numerically shown in Section 6 .

Remark 4. The definition proposed here does not automatically detect if a problem is actually one dimensional. In fact, this seems to be impossible for a linear method like those considered herein, among other things because this depends on the boundary conditions of the problem. We could consider a nonlinear method and a natural choice would be to take $\mathbf{k}$ in the direction of the gradient of the unknown in the reference domain $\nabla_{\xi} u_{h}$, that is to say,

$\mathbf{k}=\left|\nabla_{\xi} u_{h}\right|^{-1} \nabla_{\xi} u_{h}$.

We have checked that this method detects the situation of Example 3 in Section 4 but does not in general improve the results respect to those obtained using (19) and the convergence of the fixed point scheme to deal with the nonlinearity is quite hard when anisotropy is important. In any case, if a nonlinear method is to be considered it seems to be much more reasonable to introduce a shock capturing term adding crosswind diffusion like the one proposed in [11].

\section{Numerical examples}

In this section we present numerical examples illustrating the behavior of the method proposed. The first two of them illustrate the behavior of the method on anisotropic meshes, the third one shows the importance of satisfying the restriction imposed by the error analysis when elements of order higher than one are used and the last two how the method behaves on isotropic but unstructured meshes. 


\subsection{Convection-diffusion with anisotropic refinement}

In this subsection we consider a convection-diffusion problem $(s=0)$ on the domain $\Omega=[0,1] \times[0,1]$ with zero Dirichlet boundary conditions on $\partial \Omega$ and a force $f=1$. We consider a diffusion coefficient $\varepsilon_{i j}=\varepsilon \delta_{i j}$, where $\varepsilon=10^{-4}$, and three different velocities:

1. $\mathbf{a}=(1,0)$,

2. $\mathbf{a}=(0,1)$,

3. $\mathbf{a}=(\sqrt{2} / 2, \sqrt{2} / 2)$.
A reference solution of this problem was found using a mesh of $100 \times 100$ elements refined according to the velocity. In case 1 it was refined in the direction $x$ near the right wall and was uniform in the direction $y$; in the second case it was uniform in the direction $x$ and refined in the direction $y$ near the upper wall and in the third case it was refined in both directions near the right and upper walls. The smallest element size is about $2.5 \times 10^{-5}$. The results for the three cases are shown in Fig. 3. Note the presence of a strong boundary layer on the right wall in case 1 , on the upper wall in case 2 and on both the upper and right walls in case 3 .
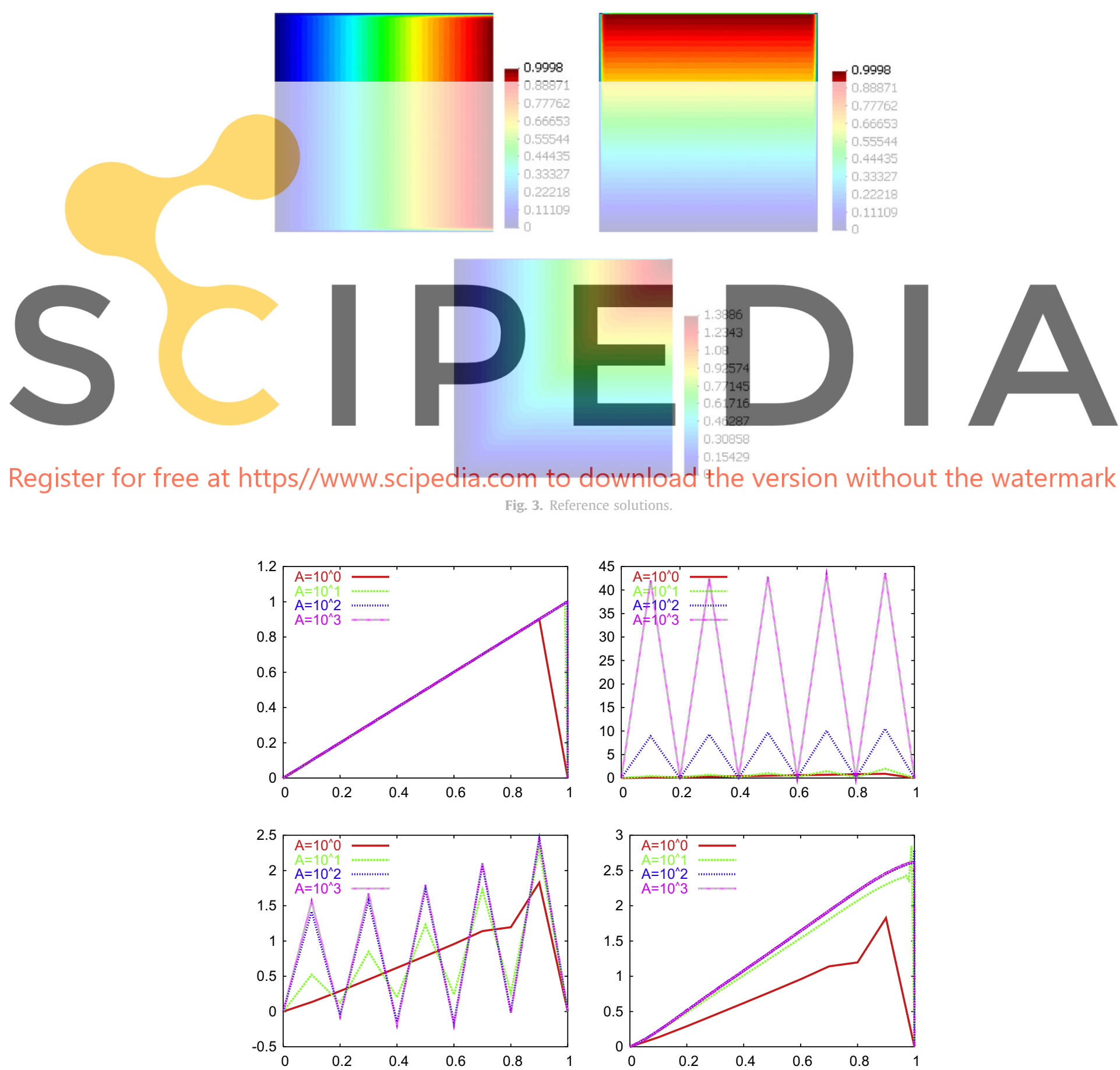

Fig. 4. Solutions obtained using (1) with $h_{\min }$ in case 1 (top left), in case 2 (top right) and in case 3 (bottom). 
For each case, the problem was also solved using a uniform mesh of $10 \times 10$ elements using the ASGS method and the definition of the stabilization parameter given by (1) taking $h$ as $h_{\text {min }}$ (the minimum element length), as $h_{\max }$ (the maximum element length) and as $h_{a}$ (the streamline element length) and also using expression (19), which is what we propose here. When the elements of the mesh are squares the definition given by (1) yields the same result taking $h$ as $h_{\min }$ or $h_{\max }$ or $h_{a}$ (when $h_{1}=h_{2}=h$ expressions (21) and (22) give $h_{\varepsilon}=h_{a}=h$ ). In these cases also expression (19) gives a similar result.
Then the behavior of the method with respect to the mesh aspect ratio was analyzed. To this end, the problem was solved using the ASGS method and meshes of $10 \times 10$ and also $100 \times 10,1000 \times 10,10,000 \times 10$ elements, giving aspect ratios $\mathrm{A}=10^{0}, 10^{1}, 10^{2}, 10^{3}$. To analyze the results we plot the unknown along the line $y=0.5$ in case 1 , along the line $x=0.5$ in case 2 and along the lines $x=0.9$ and $y=0.9$ in case 3 . The results using the stabilization parameter defined by (1) taking $h$ as $h_{\min }$ are shown in Fig. 4, those obtained taking $h$ as $h_{\max }$ are shown in Fig. 5, those obtained using $h_{a}$ are shown in Fig. 6 and those
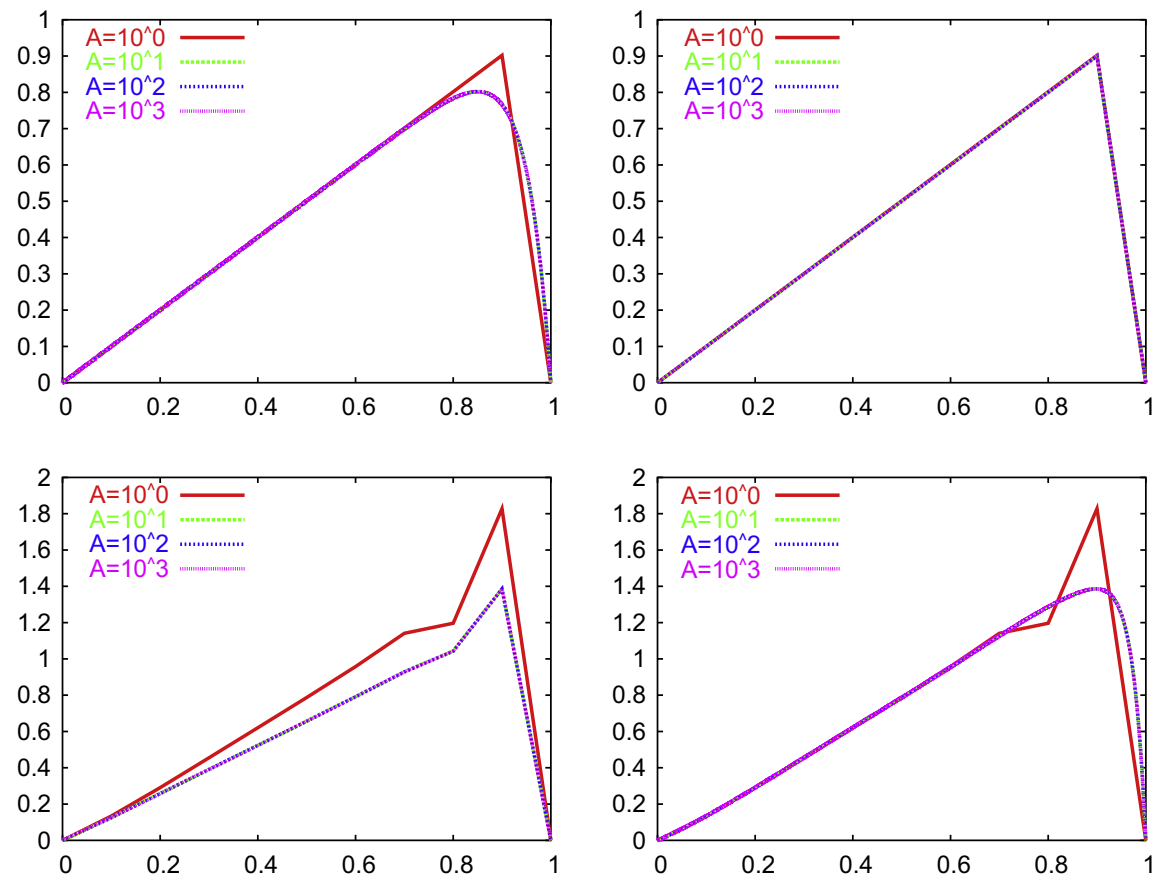

Fig. 5. Solutions obtained using (1) with $h_{\max }$ in case 1 (top left), in case 2 (top right) and in case 3 (bottom).
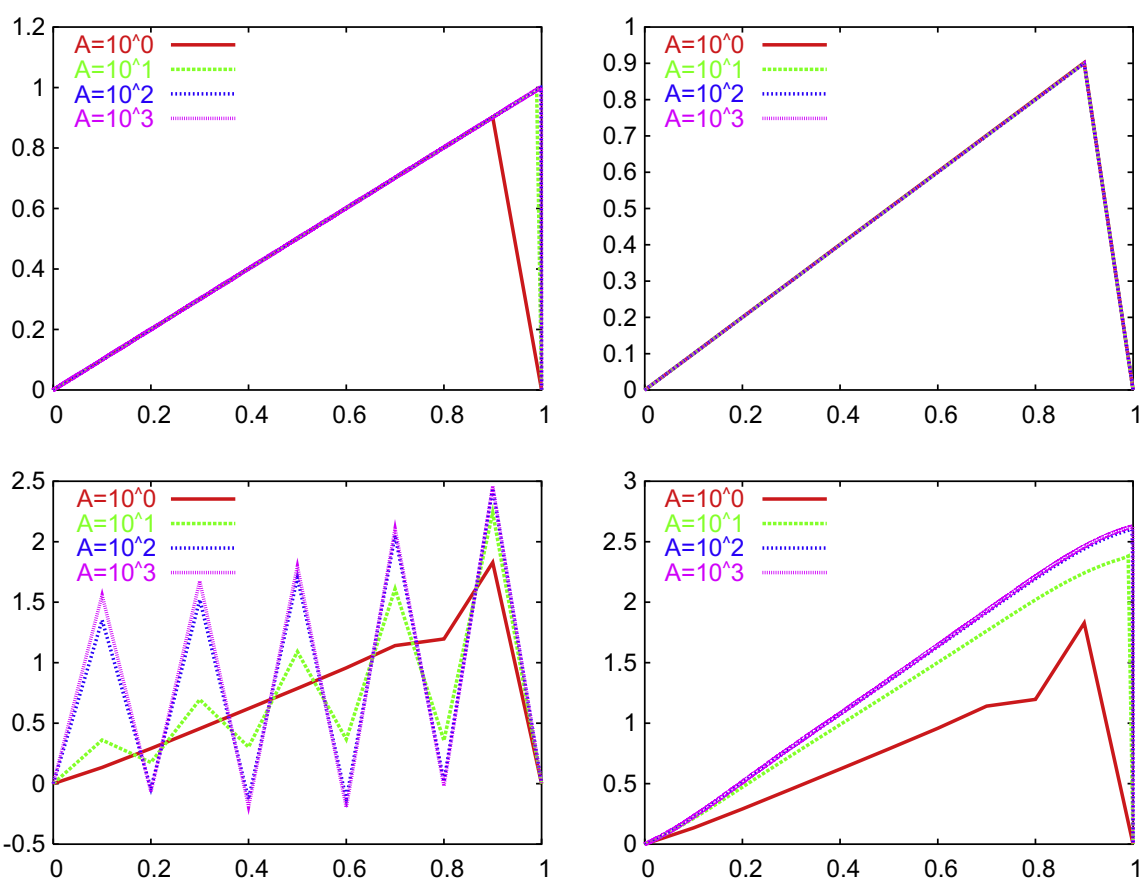

Fig. 6. Solutions obtained using (1) with $h_{a}$ in case 1 (top left), in case 2 (top right) and in case 3 (bottom). 
obtained using the stabilization parameter defined by (19) are shown in Fig. 7.

The minimum requirement we should pose to evaluate the behavior of a method is that the solution obtained using any anisotropic grid cannot be worse than the solution obtained using the $10 \times 10$ grid, or in other words, we should require that the solution cannot get worse when the dimension of the finite element space is increased in a nested way. This is what happens if we use the stabilization parameter defined by (1) taking $h$ as $h_{\min }$ or as $h_{a}$. In the first case, the solution obtained in case 1 is improved but in cases 2 and 3 numerical oscillations appear when the stretching factor increases. In the second case the solution obtained in cases 1 and 2 is improved but in case 3 numerical oscillations appear when the mesh is anisotropically refined. On the other hand, the solution obtained using the stabilization parameter defined by (1) taking $h$ as $h_{\max }$ or the solution obtained using the expression (19) satisfy this requirement, and give similar results. In case 1 , they do not take advantage of the new points added in the direction $x$ even if the solution has a boundary layer on the right wall. This behavior is explained in Section 5 based to the error analysis of the method. It
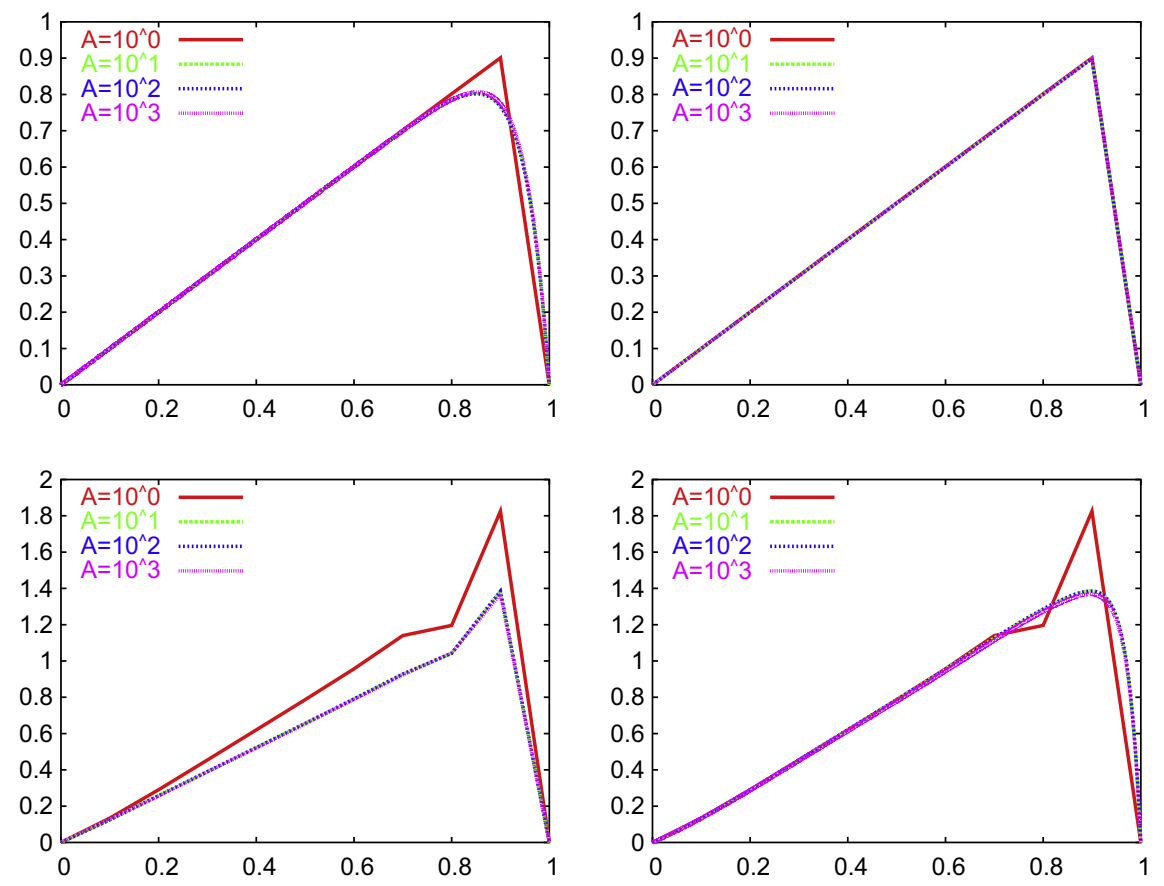

Fig. 7. Solutions obtained using (19) in case 1 (top left), in case 2 (top right) and in case 3 (bottom).
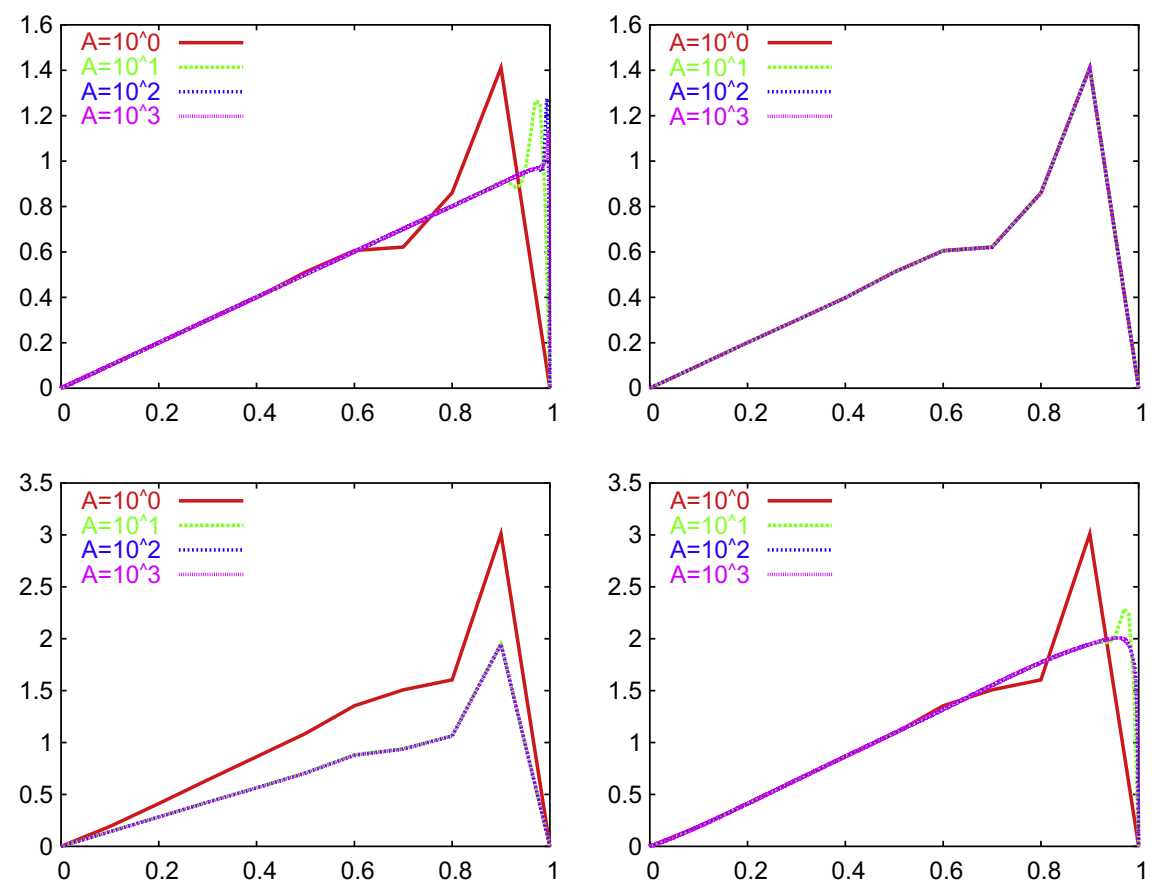

Fig. 8. Solutions obtained using the OSS method and (1) with $h_{\max }$ in case 1 (top left), in case 2 (top right) and in case 3 (bottom). 
was also shown that the OSS method does not have this problem. This can be seen in Fig. 8 where results obtained using the stabilization parameter defined by (1) taking $h$ as $h_{\max }$ are shown.

Let us finally remark that in some cases the solution obtained using (1) taking $h$ as $h_{\min }$ or as $h_{a}$ can give a better solution than the method proposed here, as occurs in case 1 , even if they present numerical oscillations in other cases.
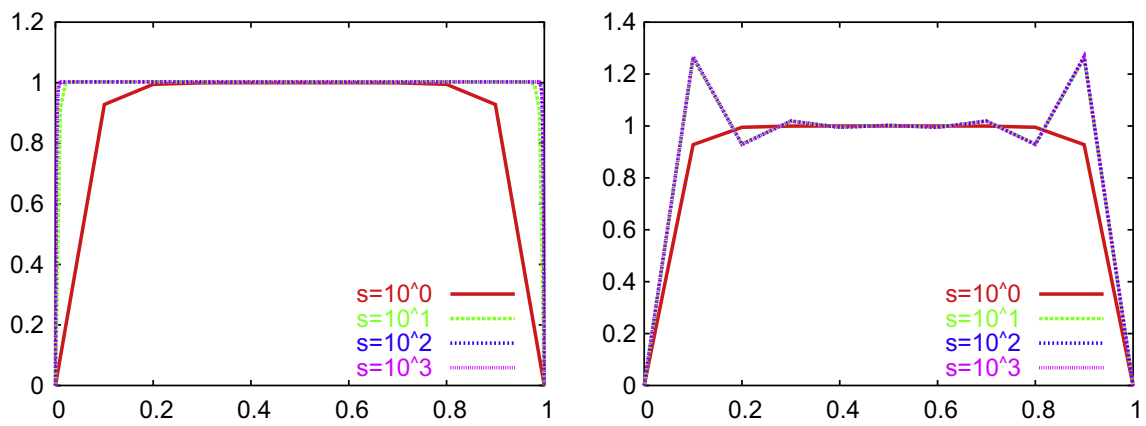

Fig. 9. Solution obtained using (1) with $h_{\min }$ along the lines $y=0.5$ and $x=0.5$.
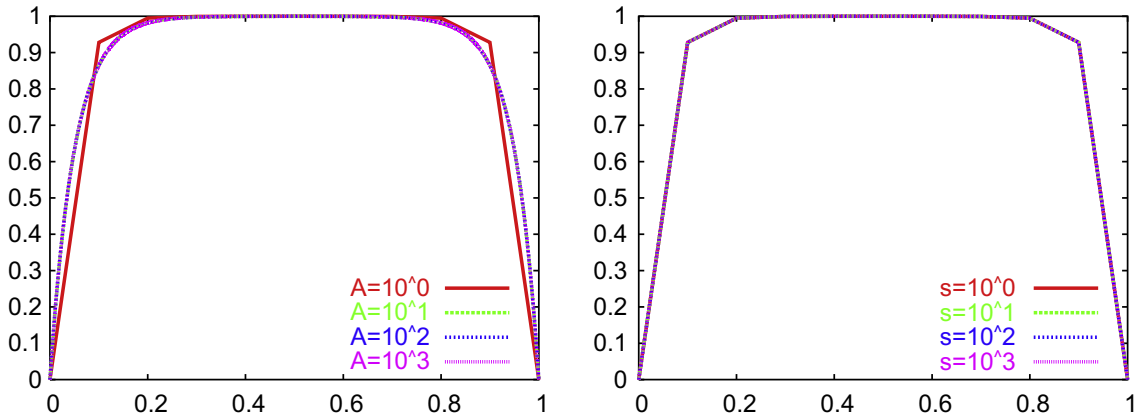

Fig. 10. Solution obtained using (1) with $h_{\max }$ along the lines $x=0.5$ and $y=0.5$.
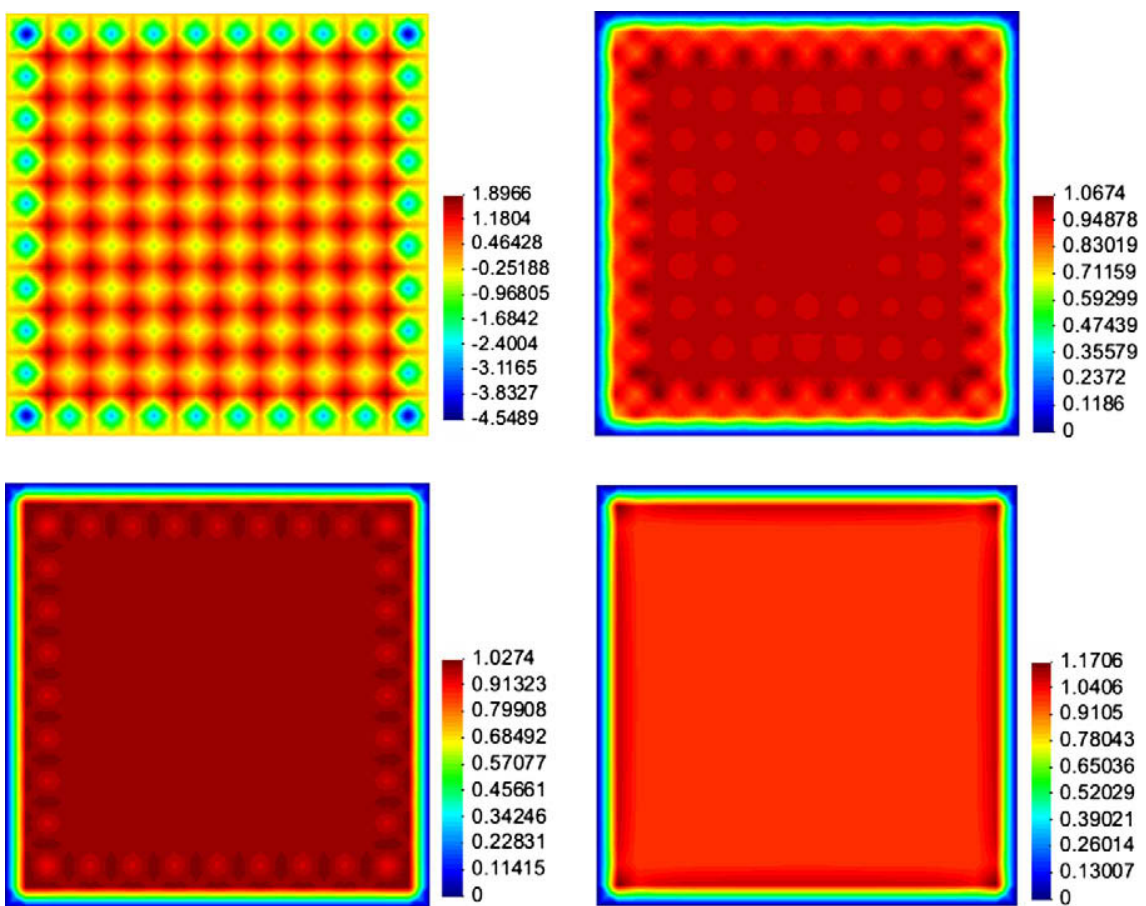

Fig. 11. Solution to the Poisson problem obtained using (1) with $c_{1}=16$ (top left), with $c_{1}=24$ (top right), with $c_{1}=48$ (bottom left) and with $c_{1}=96$ (bottom right). 
$1000 \times 10, \quad 10,000 \times 10$ elements, giving aspect ratios $\mathrm{A}=10^{0}, 10^{1}, 10^{2}, 10^{3}$. To analyze the results, we plot the unknown along the line $y=0.5$ and along the line $x=0.5$. In this case, the results obtained using the stabilization parameter defined by (1) taking $h$ as $h_{\max }$ and those obtained using (19) are the same. Therefore, we compare the results obtained using (1) taking $h$ as $h_{\min }$ shown in Fig. 9 to those obtained taking $h$ as $h_{\max }$ shown in Fig. 10.

As in the convection-diffusion problem shown in the previous subsection, the result obtained using (1) taking $h$ as $h_{\min }$ shows numerical oscillations when the mesh is anisotropically refined, whereas the results obtained using (1) taking $h$ as $h_{\max }$ do not change.

\subsection{The Poisson problem using quadratic elements}

In this subsection we consider a pure diffusive Poisson problem, which corresponds to the CDR problem in the limit of vanishing convection and reaction. The domain considered is $\Omega=[0,1] \times[0,1]$ and zero Dirichlet boundary conditions on $\partial \Omega$ are prescribed. In order to activate instabilities we introduce a forcing term that gives a solution of the form:

$u(x, y)=\left(1+\mathrm{e}^{-\alpha}-\mathrm{e}^{-\alpha x}-\mathrm{e}^{\alpha(x-1)}\right)\left(1+\mathrm{e}^{-\alpha}-\mathrm{e}^{-\alpha y}-\mathrm{e}^{\alpha(y-1)}\right)$,

which presents boundary layers on the domain boundary whose width can be controlled using the parameter $\alpha$. We solve the problem using the ASGS method and a uniform mesh of $10 \times 10$ biquadratic elements and expression (1) for different values of $c_{1}$. The results are shown in Fig. 11.

For biquadratic elements $C_{\text {inv }}=24$ [32] and it can be observed that when condition (24) is not satisfied numerical oscillations appear. Note that the Galerkin method is recovered when $c_{1} \rightarrow \infty$. It is also possible to define the constant $c_{1}$ in terms of $C_{\text {inv }}$ which, in turn, can be estimated solving local eigenvalue problems [31]. Doing that, quadratic elements are shown to be much less sensitive to the definition of the element length according to the numerical experiments presented in [31] in the context of the Navier-Stokes problem.

\subsection{A convection-diffusion-reaction problem on isotropic meshes}

In this subsection we consider a convection-diffusion problem on the domain $\Omega=[0,1] \times[0,1]$ with zero Dirichlet boundary conditions on $\partial \Omega$ and a force $f=20$. The equation coefficients are $\varepsilon_{i j}=\varepsilon \delta_{i j}$, with $\varepsilon=10^{-2}, s=20$ and $\mathbf{a}=(3,2)$. We solve the problem on different meshes:

- Case 1: structured triangular elements of size 0.1 tilted to the right $/$.

- Case 2: structured triangular elements of size 0.1 titled to the left $\backslash$.

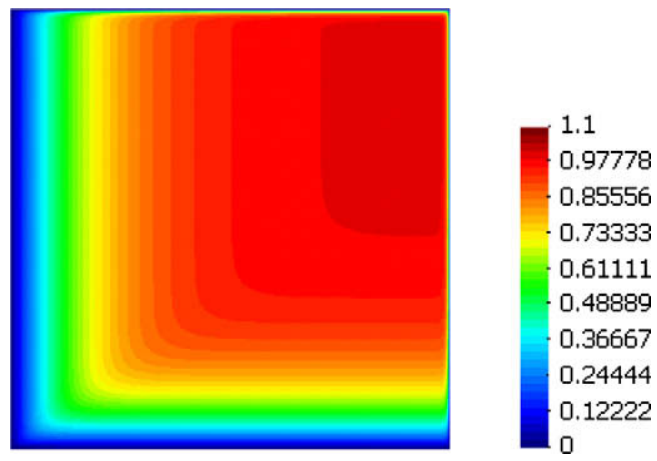

Fig. 12. Reference solution.
- Case 3: unstructured triangular elements of size 0.1 .

- Case 4: structured square elements of size 0.1.

- Case 5: unstructured square elements of sizes 0.1.

In any of these cases the mesh size is around 0.1 but it varies slightly according to the mesh design. In the case of triangular elements the element lengths are calculated as $h_{1}=J_{1 k}^{-t} J_{1 k}^{-t}$ and $h_{2}=J_{2 k}^{-t} J_{2 k}^{-t}$. The dimensionless numbers of the problem are given by

$\mathrm{D}=\frac{s h^{2}}{4 \varepsilon}=5, \quad \mathrm{P}=\frac{a h}{2 \varepsilon} \sim 18$.

A reference solution was computed on a $200 \times 200$ mesh, for which these numbers are

$\mathrm{D}=0.0125, \quad \mathrm{P}=0.9$.

The result is shown in Fig. 12. The maximum value obtained is $u_{\max }=0.99807$.

For each of the cases considered we compare the results obtained using the ASGS method and four possible definitions of the stabilization parameter. The first three are given by (1) taking the length $h$ as the minimum, the maximum and the length in the velocity direction. The fourth is the definition given by (19). Table 1 shows the maximum values obtained for each case and method. We remark that the method cannot guarantee the satisfaction of the discrete maximum principle as it is linear and therefore local

Table 1

Maximum values obtained.

\begin{tabular}{llllll}
\hline & $\begin{array}{l}\text { Case 1 } \\
(\mathrm{ST} /)\end{array}$ & $\begin{array}{l}\text { Case 2 } \\
(\mathrm{ST} \backslash)\end{array}$ & $\begin{array}{l}\text { Case 3 } \\
(\mathrm{UT})\end{array}$ & $\begin{array}{l}\text { Case 4 } \\
(\mathrm{SQ})\end{array}$ & $\begin{array}{l}\text { Case 5 } \\
(\mathrm{UQ})\end{array}$ \\
\hline (1) using $h_{\min }$ & 1.3841 & 1.2712 & 1.2052 & 1.2973 & 1.3543 \\
(1) using $h_{\max }$ & 1.1915 & 1.1486 & 1.1318 & 1.2973 & 1.2332 \\
$(1)$ using $h_{a}$ & 1.0281 & 1.3154 & 1.2437 & 1.2973 & 1.4191 \\
$(19)$ & 0.9639 & 0.9762 & 0.9773 & 1.0828 & 1.0248 \\
\hline
\end{tabular}
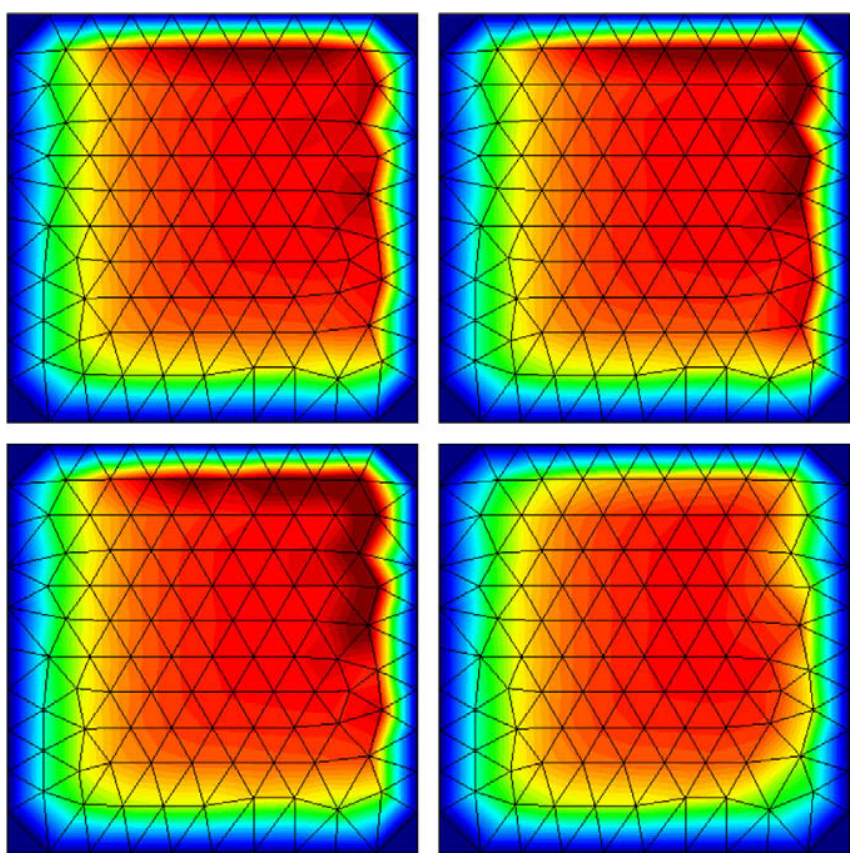

Fig. 13. Results obtained in case 3 using (1) with $h_{\max }$ (top left), with $h_{\min }$ (top right), with $h_{a}$ (bottom left) and using (19) (bottom right). 

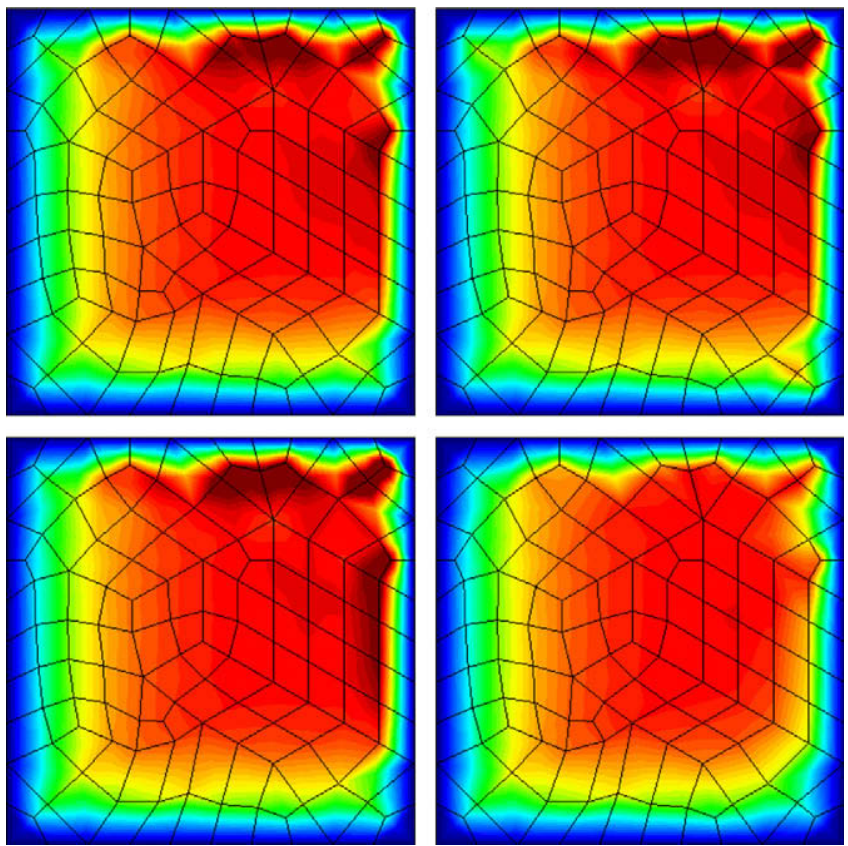

Fig. 14. Results obtained in case 5 using (1) with $h_{\max }$ (top left), with $h_{\min }$ (top right), with $h_{a}$ (bottom left) and using (19) (bottom right).

Table 2

Maximum values obtained.

\begin{tabular}{llllll}
\hline & $\begin{array}{l}\text { Case 1 } \\
(\mathrm{ST} /)\end{array}$ & $\begin{array}{l}\text { Case 2 } \\
(\mathrm{ST} \backslash)\end{array}$ & $\begin{array}{l}\text { Case 3 } \\
(\mathrm{UT})\end{array}$ & $\begin{array}{l}\text { Case 4 } \\
(\mathrm{SQ})\end{array}$ & $\begin{array}{l}\text { Case 5 } \\
\text { (UQ) }\end{array}$ \\
\hline (1) using $h_{\min }$ & 1.6557 & 1.5603 & 1.3724 & 1.5434 & 1.5624 \\
$(1)$ using $h_{\max }$ & 1.3170 & 1.4360 & 1.2937 & 1.5434 & 1.3779 \\
$(1)$ using $h_{a}$ & 1.7511 & 1.2303 & 1.4096 & 1.5434 & 1.6603 \\
$(19)$ & 1.1453 & 1.2287 & 1.2213 & 1.5434 & 1.2536 \\
\hline
\end{tabular}

Table 3

Minimum values obtained.

\begin{tabular}{llllll}
\hline & $\begin{array}{l}\text { Case 1 } \\
(\mathrm{ST} /)\end{array}$ & $\begin{array}{l}\text { Case 2 } \\
(\mathrm{ST} \backslash)\end{array}$ & $\begin{array}{l}\text { Case 3 } \\
(\mathrm{UT})\end{array}$ & $\begin{array}{l}\text { Case 4 } \\
(\mathrm{SQ})\end{array}$ & $\begin{array}{l}\text { Case 5 } \\
(\mathrm{UQ})\end{array}$ \\
\hline (1) using $h_{\min }$ & -0.0387 & -0.0377 & -0.0467 & -0.0479 & -0.0788 \\
(1) using $h_{\max }$ & -0.0345 & -0.0363 & -0.0408 & -0.0479 & -0.0717 \\
$(1)$ using $h_{a}$ & -0.0415 & -0.0331 & -0.0503 & -0.0479 & -0.0805 \\
$(19)$ & -0.0272 & -0.0331 & -0.0451 & -0.0479 & -0.0743 \\
\hline
\end{tabular}

oscillations could appear, although the results are excellent using expression (19).

Figs. 13 and 14 show the results obtained using the four definitions considered on unstructured meshes (cases 3 and 5), all given in the same scale as Fig. 12.

\subsection{Skew advection on isotropic meshes}

In this subsection we consider the problem of skew advection on the domain $\Omega=[0,1] \times[0,1]$. Boundary conditions are set to one in $x=0, y>0.5$ and $y=1$ and zero in the rest of $\partial \Omega$. The equation coefficients are $\varepsilon_{i j}=\varepsilon \delta_{i j}$, with $\varepsilon=10^{-6}, s=0$ and $\mathbf{a}=(2,-3)$ and the forcing term is $f=0$. We solve the problem using the ASGS method and the same meshes of the previous example but using a mesh size of 0.05 . As in the previous example, for each of the cases considered we compare the results obtained using the multiscale formulation using four possible definitions of the stabilization parameter. The first three are given by (1) taking the length $h$ as the minimum, the maximum and the length in the velocity direction. The fourth is the definition given by (19). Table 2 shows the maximum values (which should be one) and Table 3 shows the minimum values (which should be zero) obtained for each case and method.

Figs. 15 and 16 show the results obtained using the four definitions considered on unstructured meshes (cases 3 and 5), all given in the same scale as Fig. 12.
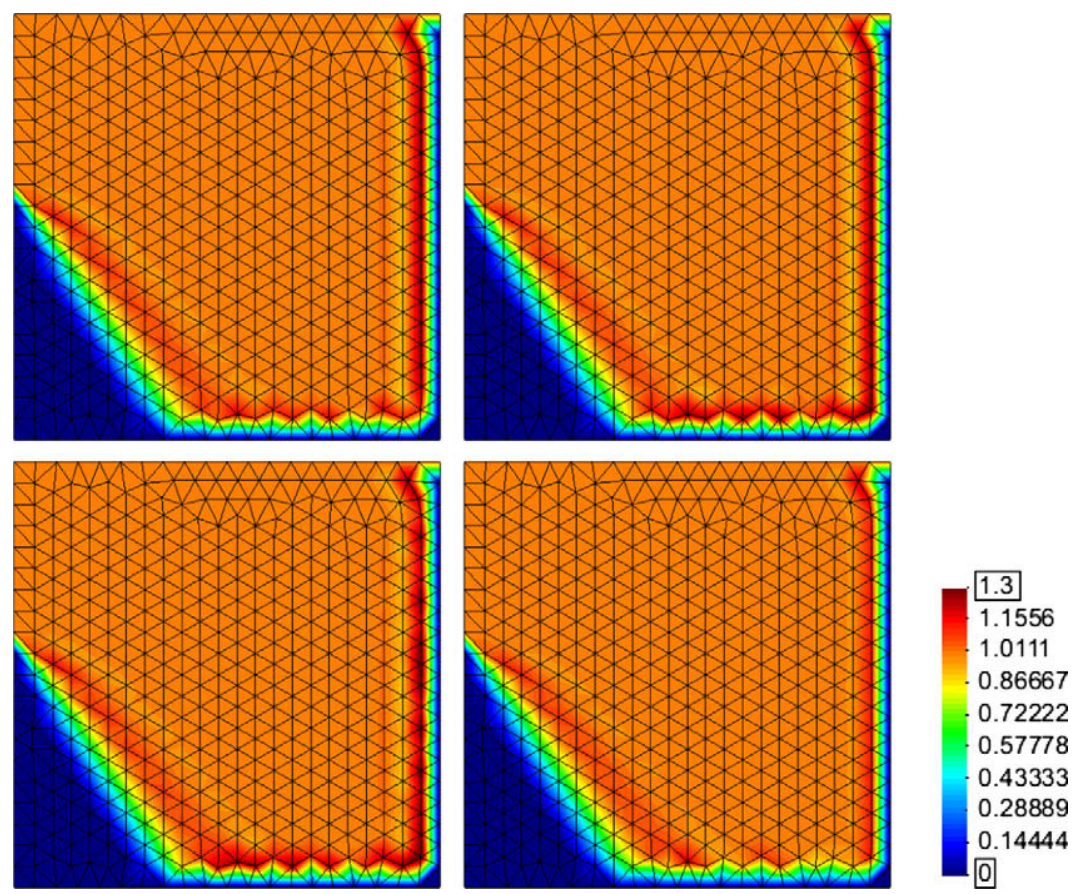

Fig. 15. Results obtained in case 3 using (1) with $h_{\max }$ (top left), with $h_{\min }$ (top right), with $h_{a}$ (bottom left) and using (19) (bottom right). 

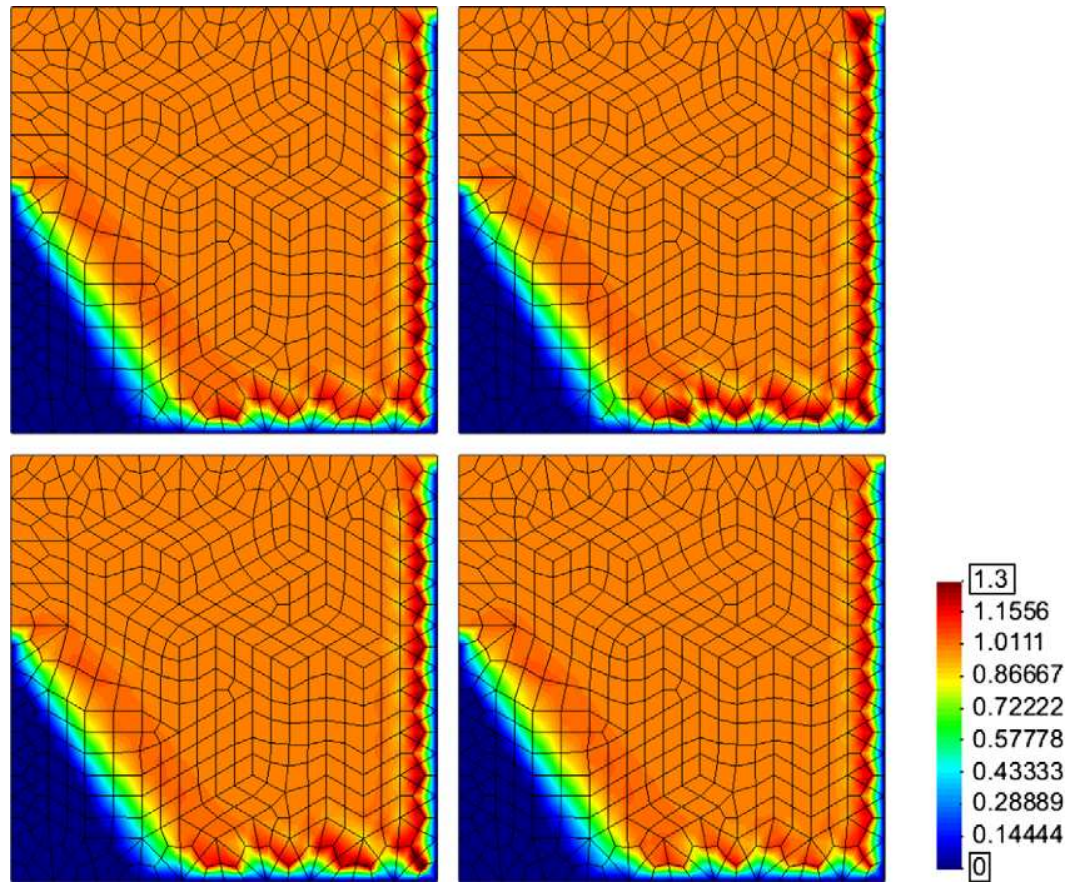

Fig. 16. Results obtained in case 5 using (1) with $h_{\max }$ (top left), with $h_{\min }$ (top right), with $h_{a}$ (bottom left) and using (19) (bottom right).

\section{Conclusions}

The definition of the stabilization parameters in the case of the scalar convection-diffusion-reaction problem has been revisited. The variational multiscale method provides a natural framework to understand the problem. Starting from this point and introducing a transformation of the fine scale problem to the reference domain the dependence of the stabilization parameters on the equation coefficients and element length (through the Jacobian of such transformation) has been identified. This strategy is crucial to understand the effect of the mesh anisotropy in the stabilization parameter. A deeper inspection of the Fourier argument presented in [14] permitted to obtain an exact representation of the Green function (12) and a first approximation to it. The well-known exact solution to the one dimensional problem has been used to find the constants of the parameter in a very natural way. Finally the direction of the wave number vector $\mathbf{k}$ where instabilities appear has been defined and a new expression for the stabilization parameter has been proposed.

Based on numerical experiments using linear elements and on the error analysis, the behavior of the method resulting from different choices of the stabilization parameter has been established:

1. Expression (1) with $h=h_{\min }$ results in an unstable method in the limit of strong anisotropy, although numerical oscillations not always appear. In some cases results are optimal, as in Example 3. This is explained by the fact that the last term in (26) vanishes in the limit of strong anisotropy.

2. Expression (1) with $h=h_{\max }$ results in a stable method that may be overdiffusive in some cases when the ASGS method is used. This happens in Example 3 and is explained by the fact that the last term in (26) does not vanish in the limit of strong anisotropy. By contrast, when the OSS method is used optimal results are obtained.

3. Expression (1) with $h=h_{a}$ behaves as (1) with $h_{\min }$ in the limit of strong anisotropy.

4. Expression (19) behaves as (1) with $h=h_{\max }$ in the limit of strong anisotropy.
5. Expression (19) gives excellent results in isotropic unstructured meshes, significantly better than the other three. Obviously the method cannot guarantee the satisfaction of the discrete maximum principle as it is linear and therefore local oscillations could appear.

When higher order elements are used condition (24) must be satisfied for the error analysis to be valid. This is confirmed by the results obtained using uniform meshes to solve the Poisson problem, which present numerical oscillations if (24) is not satisfied. The behavior of the different methods under anisotropic refinement is a much harder question.

\section{Acknowledgment}

J. Principe was partially supported by the Spanish Project OPTIDIS (ENE2005-05274) which is gratefully acknowledged.

\section{References}

[1] M. Ainsworth, J.T. Oden, A posteriori error estimation in finite element analysis, Pure and Applied Mathematics, A Wiley-Interscience Series of Texts, Monographs, and Tracts, John Wiley \& Sons, 2000.

[2] T. Apel, Anisotropic finite elements: local estimates and applications, Advances in Numerical Mathematics, Teubner, Stuttgart, 1999.

[3] C. Baiocchi, F. Brezzi, L.P. Franca, Virtual bubbles and Galerkin/least-squares type methods (Ga.L.S), Comput. Methods Appl. Mech. Engrg. 105 (1993) 125-141.

[4] J. Blasco, An anisotropic GLS-stabilized finite element method for incompressible flow problems, Comput. Methods Appl. Mech. Engrg. 197 (2008) 3712-3723.

[5] F. Brezzi, M.O. Bristeau, L. Franca, M. Mallet, G. Rogé, A relationship between stabilized finite element methods and the Galerkin method with bubble functions, Comput. Methods Appl. Mech. Engrg. 96 (1992) 117-129.

[6] F. Brezzi, L.P. Franca, T.J.R. Hughes, A. Russo, $b=\int g$, Comput. Methods Appl. Mech. Engrg. 145 (3-4) (1997) 329-339.

[7] F. Brezzi, A. Russo, Choosing bubbles for advection diffusion problems, Math. Mod. Methods Appl. Sci. 4 (4) (1994) 571-587.

[8] A.N. Brooks, T.J.R. Hughes, Streamline upwind/Petrov-Galerkin formulations for convection dominated flows with particular emphasis on the incompressible Navier-Stokes equations, Comput. Methods Appl. Mech. Engrg. 32 (1982) 199-259.

[9] V.M. Calo, Residual based multiscale turbulence modeling: finite volume simulations of bypass transition, PhD Thesis, Department of Civil and Environmental Engineering, Stanford University, 2004. 
[10] A. Cangiani, E. Süli, The residual-free-bubble finite element method on anisotropic partitions, SIAM J. Numer. Anal. 45 (4) (2007) 1654-1678.

[11] R. Codina, A discontinuity-capturing crosswind-dissipation for the finite element solution of the convection-diffusion equation, Comput. Methods Appl. Mech. Engrg. 110 (1993) 325-342.

[12] R. Codina, Comparison of some finite element methods for solving the diffusion-convection-reaction equation, Comput. Methods Appl. Mech. Engrg. 156 (1998) 185-210.

[13] R. Codina, A stabilized finite element method for generalized stationary incompressible flows, Comput. Methods Appl. Mech. Engrg. 190 (20-21) (2001) 2681-2706. Feb.

[14] R. Codina, Stabilized finite element approximation of transient incompressible flows using orthogonal subscales, Comput. Methods Appl. Mech. Engrg. 191 (39-40) (2002) 4295-4321. Aug.

[15] R. Codina, Analysis of a stabilized finite element approximation of the Oseen equations using orthogonal subscales, Appl. Numer. Math. 58 (2008) 264-283.

[16] R. Codina, E. Oñate, M. Cervera, The intrinsic time for the streamline upwind/ Petrov-Galerkin formulation using quadratic elements, Comput. Methods Appl. Mech. Engrg. 94 (1992) 239-262.

[17] R. Codina, J. Principe, J. Baiges, Subscales on the element boundaries in the variational two-scale finite element method, Comput. Methods Appl. Mech. Engrg. 198 (5-8) (2009) 838-852.

[18] R. Codina, O. Soto, Approximation of the incompressible Navier-Stokes equations using orthogonal subscale stabilization and pressure segregation on anisotropic finite element meshes, Comput. Methods Appl. Mech. Engrg. 193 (15-16) (2004) 1403-1419. Apr.

[19] J. Donea, A Taylor-Galerkin method for convection transport problems, Int. J. Numer. Methods Engrg. 20 (1984) 101-119.

[20] J. Douglas, T. Russel, Numerical methods for convection dominated problems based on combining the method of characteristics with finite elements or finite difference procedures, SIAM J. Numer. Anal. 19 (1982) 871-885.

[21] J. Douglas, J. Wang, An absolutely stabilized finite element method for the Stokes problem, Math. Comput. 52 (1989) 495-508.

[22] A.H. ElSheikh, S.E. Chidiac, W.S. Smith, A posteriori error estimation based on numerical realization of the variational multiscale method, Comput. Methods Appl. Mech. Engrg. 197 (45-48) (2008) 3637-3656.

[23] L. Formaggia, S. Micheletti, S. Perotto, Anisotropic mesh adaptation in computational fluid dynamics: application to the advection-diffusionreaction and the Stokes problems, Appl. Numer. Math. 51 (4) (2004) 511-533.

[24] L. Franca, C. Farhat, Bubble functions prompt unusual stabilized finite element methods, Comput. Methods Appl. Mech. Engrg. 123 (1995) 299-308.

[25] L. Franca, S.L. Frey, T.J.R. Hughes, Stabilized finite element methods: I. Application to the advective-diffusive model, Comput. Methods Appl. Mech. Engrg. 95 (1992) 253-276.

[26] L. Franca, G. Hauke, A. Masud, Revisiting stabilized finite element methods for the advective-diffusive equation, Comput. Methods Appl. Mech. Engrg. 195 (13-16) (2006) 1560-1572.

[27] L. Franca, A.P. Macedo, A two-level finite element method and its application to the Helmholtz equation, Int. J. Numer. Methods Engrg. 43 (1998) 23-32.

[28] L. Franca, A. Nesliturk, M. Stynes, On the stability of residual-free bubbles for convection-diffusion problems and their approximation by a two-level finite element method, Comput. Methods Appl. Mech. Engrg. 166 (1-2) (1998) 3549.

[29] L. Franca, A. Russo, Deriving upwinding, mass lumping and selective reduced integration by residual-free bubbles, Appl. Math. Lett. 9 (5) (1996) 253-276.

[30] L. Franca, F. Valentin, On an improved unusual stabilized finite element method for the advective-reactive-diffusive equation, Comput. Methods Appl. Mech. Engrg. 190 (13-14) (2000) 1785-1800.

[31] Ch. Förster, W.A. Wall, E. Ramm, Stabilized finite element formulation for incompressible flow on distorted meshes, Int. J. Numer. Methods Fluids 60 (10) (2008) 1103-1126.
32] I. Harari, T.J.R. Hughes, What are $\mathrm{c}$ and h?: inequalities for the analysis and design of finite element methods, Comput. Methods Appl. Mech. Engrg. 97 (2) (1992) 157-192

[33] G. Hauke, A simple subgrid scale stabilized method for the advectiondiffusion-reaction equation, Comput. Methods Appl. Mech. Engrg. 191 (27-28) (2002) 2925-2947.

[34] G. Hauke, M.H. Doweidar, M. Miana, The multiscale approach to erro estimation and adaptivity, Comput. Methods Appl. Mech. Engrg. 195 (13-16) (2006) 1573-1593.

[35] G. Hauke, D. Fuster, M.H. Doweidar, Variational multiscale a-posteriori error estimation for multi-dimensional transport problems, Comput. Methods Appl. Mech. Engrg. 197 (2008) 2701-2718.

[36] G. Hauke, A. Garcia-Olivares, Variational subgrid scale formulations for the advection-diffusion-reaction equation, Comput. Methods Appl. Mech. Engrg. 190 (51-52) (2001) 6847-6865.

[37] T.J.R. Hughes, Multiscale phenomena, Green's functions, the Dirichlet-toNeuman formulation, subgrid scale models, bubbles and the origins of stabilized methods, Comput. Methods Appl. Mech. Engrg. 127 (1995) 387-401.

38] T.J. Hughes, A.N. Brooks, A multidimensional upwind scheme with no crosswind diffusion, in: T.J.R. Hughes (Ed.), FEM for Convection Dominated Flows, ASME, New York, 1979.

[39] T.J.R. Hughes, G.R. Feijóo, L. Mazzei, J.B. Quincy, The variational multiscale method - a paradigm for computational mechanics, Comput. Methods Appl. Mech. Engrg. 166 (1998) 3-24.

[40] T.J.R. Hughes, L.P. Franca, G.M. Hulbert, A new finite element formulation for computational fluid dynamics: VIII. The Galerkin/least-squares method for advective-diffusive equations, Comput. Methods Appl. Mech. Engrg. 73 (2) (1989) 173-189.

[41] T.J.R. Hughes, M. Mallet, A new finite element formulation for computational fluid dynamics: III. The generalized streamline operator for multidimensional advective-diffusive systems, Comput. Methods Appl. Mech. Engrg. 58 (3) (1986) 305-328.

[42] C. Johnson, U. Nävert, J. Pitkäranta, Finite element methods for linear hyperbolic equations, Comput. Methods Appl. Mech. Engrg. 45 (1984) 285312

[43] D.W. Kelly, S. Nakazawa, O.C. Zienkiewicz, J.C. Heinrich, A note on upwinding and anisotropic balancing dissipation in finite element approximations to convective diffusion problems, Int. J. Numer. Methods Engrg. 15 (1980) 17051711 .

[44] S. Mittal, On the performance of high aspect ratio elements for incompressible flows, Comput. Methods Appl. Mech. Engrg. 188 (2000) 269-287.

[45] E. Oñate, Derivation of stabilized equations for numerical solution of advective-diffusive transport and fluid flow problems, Comput. Methods Appl. Mech. Engrg. 151 (1-2) (1998) 233-265.

[46] E. Oñate, F. Zarate, S.R. Idelsohn, Finite element formulation for convectivediffusive problems with sharp gradients using finite calculus, Comput Methods Appl. Mech. Engrg. 195 (13-16) (2006) 1793-1825.

[47] F. Shakib, T.J.R. Hughes, Z. Johan, A new finite element formulation for computational fluid dynamics: X. The compressible Euler and Navier-Stokes equations, Comput. Methods Appl. Mech. Engrg. 89 (1-3) (1991) 141-219.

[48] C.A. Taylor, T.J.R. Hughes, C.K. Zarins, Finite element modeling of blood flow in arteries, Comp. Methods Appl. Mech. Engrg. 158 (1-2) (1998) 155-196.

[49] T.E. Tezduyar, Computation of moving boundaries and interfaces and stabilization parameters, Int. J. Numer. Methods Fluids 43 (2003) 555-575.

[50] T.E. Tezduyar, S. Mittal, S.E. Ray, R. Shih, Incompressible flow computations with stabilized bilinear and linear equal-order-interpolation velocity-pressure elements, Comput. Methods Appl. Mech. Engrg. 95 (2) (1992) 221-242.

[51] C.H. Whiting, K.E. Jansen, A stabilized finite element method for the incompressible Navier-Stokes equations using a hierarchical basis, Int. J. Numer. Methods Fluids 35 (1) (2001) 93-116. 